
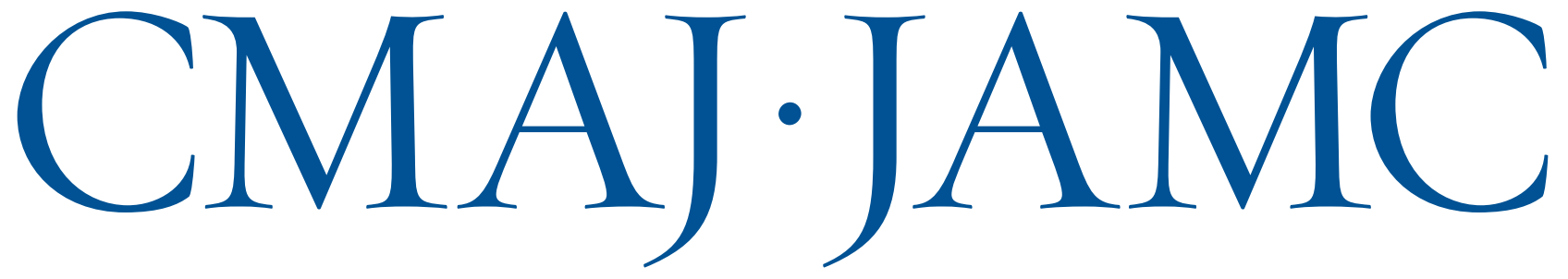

DECEMBER 2, 2008, VOLUME 179(12) • LE 2 DÉCEMBRE 2008, VOLUME 179(12)

\title{
Recommandations canadiennes pour les pratiques optimales de soins de l'AVC (mise à jour de 2008) - Sommaire
}

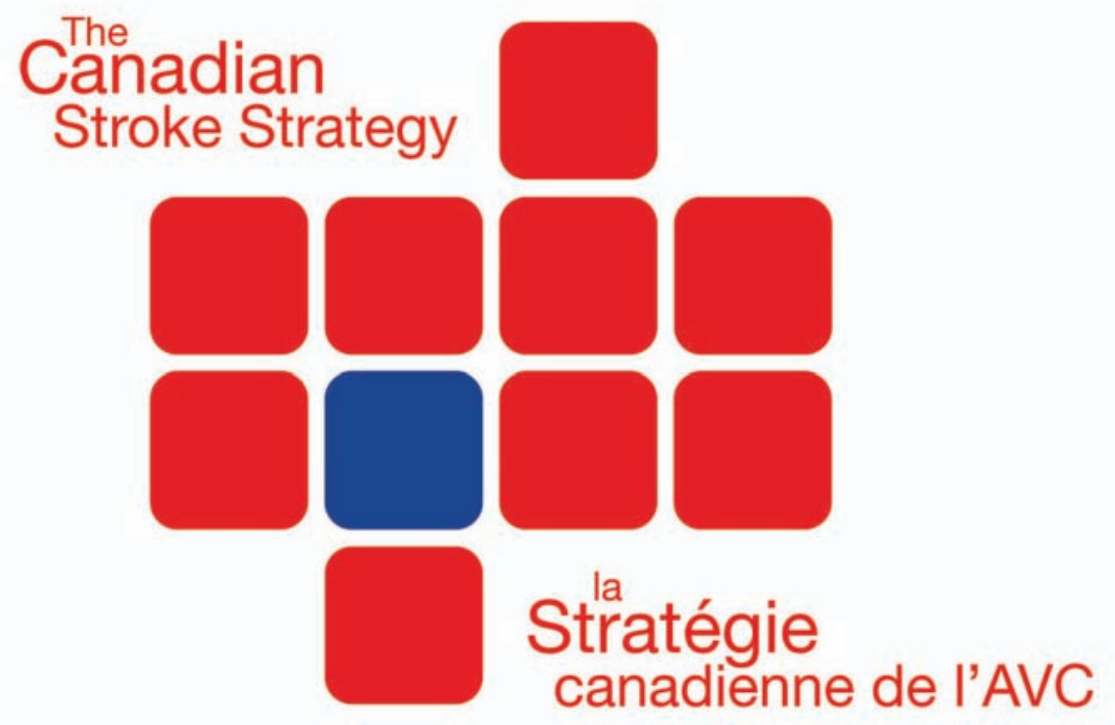

JAMC 2008;179(12 SUPPL):SF1-SF29 


\title{
Recommandations canadiennes pour les pratiques optimales de soins de l'AVC (mise à jour de 2008)
}

\author{
Patrice Lindsay BScN PhD, Mark Bayley MD, Chelsea Hellings BA, Michael Hill MSc MD, \\ Elizabeth Woodbury BCom MHA, Stephen Phillips MBBS (Groupe de rédaction, Pratiques \\ optimales et normes, au nom de la Stratégie canadienne de l'AVC, une initiative conjointe du \\ Réseau canadien contre les accidents cérébrovasculaires et de la Fondation des maladies du cœur \\ du Canada*)
}

Ce document, version abrégée des Recommandations canadiennes pour les pratiques optimales de soins de l'AVC (mise à jour de 2008) donne un aperçu du processus de rédaction et un sommaire des recommandations. On trouvera la version intégrale (y compris le glossaire, les annexes et les références), sur www.cmaj.ca/content/vol179/issue12 /\#supplement.

\section{Aperçu}

C'est à l'instigation du Réseau canadien contre les accidents cérébrovasculaires et de la Fondation des maladies du cœur du Canada qu'est née la Stratégie canadienne de l'AVC. La stratégie rassemble une multitude d'intervenants et de partenaires qui travaillent de concert à l'atteinte d'un objectif commun, c'est-à-dire, concevoir et appliquer une approche coordonnée et intégrée pour la prévention de l'AVC, son traitement, la réadaptation des patients et leur réintégration dans la communauté, dans chaque province et territoire du Canada. Un meilleur accès à des services intégrés de haute qualité et efficaces dans le contexte de l'AVC pour tous les Canadiens confirmera que la Stratégie canadienne de l'AVC constitue un exemple de réformes novatrices et positives du système de santé, tant au Canada qu'à l'échelle internationale.

La Stratégie canadienne de l'AVC propose un cadre qui facilite l'adoption à grande échelle des pratiques optimales fondées sur des preuves tout au long du continuum des soins de l'AVC, en s'attardant davantage à deux échelons :

- l'échelon national, où la création de groupes de travail visant à s'attaquer aux projets prioritaires appuie les efforts provinciaux et territoriaux grâce à la coordination, à l'élaboration des contenus et à la communication;

- l'échelon provincial et territorial, où l'application des pratiques optimales en vue de la prévention et du traitement de l'AVC, ainsi que de la réadaptation et de la réintégration des patients en milieu communautaire occupe l'avantscène au chapitre des soins de santé.

Les pratiques optimales et les normes représentent l'une des cinq plates-formes nationales prioritaires de la Stratégie canadienne de l'AVC. L'objectif de la plate-forme Pratiques optimales et normes est de transformer la prévention et les soins de l'AVC de manière à s'assurer que les pratiques optimales fondées sur des preuves sont intégrées au système de santé canadien. L'élaboration et la diffusion des Recommandations canadiennes pour les pratiques optimales de soins de
l'AVC sont un premier pas en ce sens.

Le Groupe de travail sur les pratiques optimales et les normes a été mis sur pied après que l'on a constaté que les résultats de la recherche sur l'AVC ne trouvent pas toujours écho auprès des professionnels de la santé, des administrateurs d'hôpitaux, des ministères de la Santé et, plus important encore, des personnes victimes d'AVC. Ainsi, les pratiques optimales ne sont pas appliquées de manière systématique, ce qui creuse un important fossé au chapitre de la qualité des soins de l'AVC, entre ce qui est souhaitable et ce qui se fait dans la réalité. L'objectif principal de la Stratégie canadienne de l'AVC est de contribuer à combler ce fossé. On trouvera à l'Annexe 1 la liste des membres du Groupe de travail sur les pratiques optimales et les normes.

La première édition des Recommandations canadiennes pour les pratiques optimales de soins de l'AVC, publiée en $2006^{1}$, incluait un plan permanent qui prévoyait officiellement leur mise à jour tous les deux ans, pour faire en sorte qu'elles restent actuelles et qu'elles soient en phase avec d'autres initiatives similaires à l'échelon national et international. La mise à jour de 2008 comprend des révisions apportées aux 24 recommandations de pratiques optimales publiées en 2006 et 4 nouvelles recommandations portant sur les services médicaux d'urgence, la prise en charge de l'accident ischémique transitoire et de l'AVC mineur, les soins aigus chez le patient hospitalisé et l'atteinte cognitive d'origine vasculaire.

\section{Portée, but et public cible}

Les Recommandations canadiennes pour les pratiques optimales de soins de l'AVC sont le fruit d'une analyse approfondie de la recherche internationale sur l'AVC et des recommandations ou lignes directrices sur les pratiques optimales fondées sur des preuves publiées au sujet de l'AVC. Le présent document se veut une synthèse des pratiques optimales de soins de l'AVC dans tout le continuum de soins et un cadre de référence pour les provinces et territoires qui mettent au point et appliquent des stratégies relatives à l'AVC. Dans le présent document, le terme «continuum des soins de l'AVC» se définit par les éléments suivants :

- prévention primaire, promotion de la santé et sensibilisation du public

- prise en charge de l'AVC hyperaigu

- prise en charge de l'AVC aigu

- réadaptation après l'AVC

- prévention des récidives d'AVC (prévention secondaire)

*Voir les Annexes 1 et 2 pour les affiliations du groupe de rédaction et une liste complète des comités et des autres collaborateurs qui ont participé à rédiger cette publication. 
- réintégration en milieu communautaire

- rétablissement à long terme

La mise à jour de 2008 des Recommandations canadiennes pour les pratiques optimales de soins de l'AVC présente les éléments les plus cruciaux des soins efficaces de l'AVC, qui se fondent sur les preuves et(ou) qui sont des moteurs clés du système de santé et sont pertinents dans le contexte canadien. Ces lignes directrices s'adressent aux professionnels de la santé, par l'entremise du système de soins de santé qui dessert les personnes affectées par un AVC, mais elles s'adressent également aux décideurs, aux planificateurs, aux organismes subventionnaires et aux administrateurs du système de santé.

À l'intérieur du système de soins de santé, on dénombre en général trois niveaux d'établissements qui offrent des services pour l'AVC : les centres de traitement complet pour l'AVC, les centres qui offrent un degré intermédiare de services pour l'AVC et les centres auxquels il manque les ressources nécessaires pour prodiguer les soins de l'AVC (voir la version intégrale pour une description détaillée de ces établissements et un glossaire pour la terminologie de l'AVC). C'est un fait reconnu que, faute de ressources (humaines, financières et matérielles), il est parfois difficile d'appliquer chaque recommandation de ce document. Toutefois, les recommandations sont présentées comme des «étapes normatives»vers lesquelles toutes les organisations et tous les systèmes desservant les personnes victimes d'AVC devraient tendre. De plus, il s'agit d'outils précieux que peuvent utiliser les intervenants qui militent pour l'amélioration des services de soins de l'AVC.

\section{Méthodologie : élaboration et mise à jour}

Le cadre conceptuel utilisé pour guider l'identification, la sélection, l'élaboration et la mise à jour des Recommandations canadiennes pour les pratiques optimales étaient le Practice Guideline Evaluation and Adaptation Cycle de Graham et coll'2.

\section{Direction}

Le processus d'élaboration des recommandations a été dirigé par un sous-comité du Groupe de travail sur les pratiques optimales et les normes (voir l'Annexe 1) et géré par la spécialiste du rendement et des normes du Réseau canadien contre les accidents cérébrovasculaires (P.L.). Un groupe interprofessionnel d'experts en soins de l'AVC a été formé pour participer aux groupes de travail réunis précisément pour élaborer les énoncés des recommandations canadiennes, pour chaque segment du continuum des soins de l'AVC. Le comité pour un consensus national s'est pour sa part réuni afin de fournir d'autres commentaires sur les recommandations. L'examen final du document a été confié à un groupe externe d'experts de l'AVC et d'experts en méthodologie avant sa diffusion.

Les personnes qui ont participé à l'élaboration et à la révision des lignes directrices ont été invitées à déclarer tout conflit d'intérêts potentiel par écrit. Seize personnes ont reçu des honoraires pour parler de l'AVC. On a jugé qu'aucun de ces conflits d'intérêts n'empêchait la participation impartiale des auteurs au processus de rédaction des lignes directrices. Le projet a été entièrement subventionné par la Stratégie canadienne de l'AVC, un partenariat entre le Réseau canadien contre les accidents cérébrovasculaires et la Fondation des maladies du cœur du Canada (deux organismes à but non lucratif). Ces recommandations ont été obtenues par voie de consensus entre des experts indépendants et des intervenants qui ont appliqué un processus rigoureux et les vues et intérêts de l'organisme subventionnaire n'ont aucunement influencé les recommandations finales.

\section{Détermination des sujets clés et des lignes directrices de référence de base}

Des critères ont été établis pour orienter la sélection des recommandations en vue de leur intégration dans les lignes directrices canadiennes. Ces critères ont été appliqués aux recommandations originales et à toutes les mises à jour. Les auteurs ont déterminé que pour être incluses, les recommandations devaient répondre aux critères suivants :

- être appuyées par des niveaux de preuve parmi les plus élevés et(ou) être considérées essentielles à la prestation de pratiques optimales de soins de l'AVC;

- être des moteurs de changement important du système de santé;

- concorder avec les autres recommandations canadiennes de pratiques optimales liées à l'AVC, c'est-à-dire, prise en charge de l'hypertension, du diabète et de la dyslipidémie;

- refléter dans leur totalité le continuum complet des soins de l'AVC.

Les auteurs ont convenu que toutes les recommandations seraient accompagnées d'informations précises à l'appui de leur application, c'est-à-dire, justification, exigences clés pour le système de santé, indicateurs de rendement normalisés pour en évaluer l'application et sommaire des preuves les étayant.

À l'origine, la portée et la teneur du projet ont été définies suite à une évaluation des lignes directrices et des recommandations nationales et internationales sur l'AVC, avec pour but de sélectionner les sujets à inclure dans les Recommandations canadiennes pour les pratiques optimales de soins de l'AVC $^{1,3-38}$. Les auteurs ont utilisé comme point de départ deux synthèses de lignes directrices canadiennes complètes sur les soins de l'AVC existantes, soit celles de la Canadian Stroke Quality of Care Study (CSQCS) $)^{17-20}$, qui se penchait sur les soins aigus, le télé-AVC et la prévention secondaire, et le projet SCORE (pour Stroke Canada Optimization of Rehabilitation through Evidence) $)^{38}$, qui s'attardait plus spécifiquement à la réadaptation. Ces études sur les pratiques optimales et l'évaluation du rendement dans le contexte des soins de l'AVC faisaient elles-mêmes suite à cinq réunions consensuelles (trois pour la CSQCS, une pour le projet SCORE et une mixte) tenues entre 2004 et 2006. La méthodologie rigoureuse et les conclusions détaillées de ces deux projets ont formé la base de la phase initiale de l'élaboration des Recommandations pour les pratiques optimales de soins de l'AVC. 


\section{Synthèse des recommandations pour les pratiques optimales}

Pour chaque segment du continuum des soins de l'AVC (prévention, soins des phases hyperaiguë et aiguë, réadaptation et soins en milieu communautaire), des groupes de travail d'experts se sont réunis afin de sélectionner les recommandations pertinentes à partir de la matrice ou, si nécessaire, d'en rédiger de nouvelles, fondées sur les synthèses de la littérature (voir l'Annexe 2 pour la liste des membres des groupes de travail). À la fin de l'énoncé de chaque recommandation, nous avons établi la liste des autres lignes directrices avec lesquelles ces recommandations concordent le plus, selon le cas et la pertinence (voir le Tableau 1 pour les abréviations des titres des lignes directrices utilisés dans ces listes).

Les groupes de travail ont exécuté les tâches suivantes :

- ils ont passé en revue la matrice des recommandations sur l'AVC et la documentation les étayant pour leur segment du continuum;

- ils ont passé en revue les analyses structurées de la littérature et les preuves principales à l'appui de chaque domaine lié à l'AVC;

- ils ont tenu compte des sujets additionnels appuyés par des preuves de niveau élevé, mais qui n'apparaissaient pas sur la liste initiale des sujets relevés par le groupe de travail;

- ils ont rédigé la première ébauche des recommandations pour leur segment du continuum à partir d'une sélection des lignes directrices existantes ou en les rédigeant de manière à ce qu'elles concordent avec les preuves récentes;

- ils ont fourni les références à l'appui de chaque recommandation, y compris les lignes directrices de référence centrales qui ont été adaptées ou qui ont contribué le plus à la formulation de la recommandation;

- ils ont rédigé la justification de chaque recommandation en mentionnant sa pertinence pour la prestation des soins de l'AVC;

- ils ont décrit les exigences de l'application des recommandations pour le système de soins de santé canadien;

- ils ont fourni des résumés des principales preuves tirées de la recherche étayant les recommandations.

\section{Examen des recommandations par un comité consensuel d'experts nationaux}

Une fois le travail des différents groupes complété, on a présenté à un groupe élargi d'intervenants l'ébauche des recommandations et les données les étayant aux fins de délibérations et de conclusion à l'occasion d'une réunion nationale du comité consensuel (voir la version intégrale pour la liste des participants). Le comité était formé de membres des groupes de travail, de professionnels de la santé de plusieurs disciplines intéressées par différents segments du continuum des soins de santé qui étaient indépendants du processus d'élaboration des lignes directrices, de leaders d'opinion influents et de patients ayant survécu à un AVC. Les objectifs de la réunion du comité consensuel étaient les suivants :

- discuter des mises à jour proposées aux recommandations existantes, de l'inclusion de nouvelles recommandations et autres changements suggérés au document et le modifier, si nécessaire;
- atteindre un consensus et voter sur l'ensemble des recommandations;

- débattre des stratégies d'application actuelles ou en proposer;

- prioriser les recommandations pour les pratiques optimales afin de repérer les ressources existantes en vue de leur application, tout en rappelant l'importance de toutes les recommandations dans un système intégré et coordonné de soins de l'AVC.

\section{Définition des indicateurs de rendement}

Le Groupe de travail sur l'information et l'évaluation de la Stratégie canadienne de l'AVC (voir l'Annexe 1) a été mis sur pied pour établir un cadre en vue de l'évaluation de la qualité et de la constance des soins dans tout le continuum de la prestation des soins de l'AVC. Dans le cadre de son mandat, le Groupe de travail sur l'information et l'évaluation de la Stratégie canadienne de l'AVC a passé en revue chaque recommandation finale et défini une série d'indicateurs de rendement afin de surveiller l'impact de l'application de la recommandation sur la qualité des soins aux patients et sur leur pronostic. Le groupe de travail a également préparé des «notes relatives à la mesure des indicateurs» où l'on mentionne des sources de données potentielles, des méthodes pour améliorer la collecte de données, les obstacles qui nuisent à l'accès aux données et les problématiques liées à leur qualité. On trouvera un dictionnaire complet des données à www.canadianstrokestrategy.ca.

\section{Diffusion des recommandations pour les pratiques optimales}

Après la réunion du comité consensuel, les groupes de travail se sont réunis de nouveau pour prendre connaissance des commentaires du comité consensuel, débattre des révisions suggérées et proposer la formulation définitive des recommandations. Une fois ce processus terminé, les étapes suivantes se sont succédées :

- Les recommandations et la documentation les étayant ont été passées en revue par divers experts canadiens de l'AVC indépendants et par des responsables du système de santé qui n'avaient pas participé aux étapes précédentes du processus d'élaboration des recommandations (voir la version intégrale pour une liste des examinateurs externes et une annexe qui résume les commentaires des rédacteurs du $J A M C$ et des examinateurs pairs, accompagnés de la réponse des auteurs).

- Le document a été traduit en français, et la traduction a été vérifiée par des neurologues et des infirmières bilingues spécialistes de l'AVC.

- Des mécanismes de surveillance et de rétroaction ont été mis en place pour assurer la préparation de la prochaine mise à jour.

\section{Points saillants de la mise à jour 2008}

Après la publication des Recommandations canadiennes pour les pratiques optimales de soins de l'AVC de 2006, les 
Tableau 1 : Abréviations utilisées pour citer d'autres lignes directrices et essais cliniques avec lesquels concordent les présentes recommandations*.

\begin{tabular}{|c|c|}
\hline Abréviation & Définition \\
\hline AAN & American Academy of Neurology : Report of the therapeutics and technology assessment subcommittee ${ }^{39}$ \\
\hline ACCP & American College of Chest Physicians: Evidence-based clinical practice guidelines (8th ed.) ${ }^{9}$ \\
\hline ACD & $\begin{array}{l}\text { Association canadienne du diabète : } 2008 \text { Association canadienne du diabète. Lignes directrices de pratique } \\
\text { clinique pour la prévention et le traitement du diabète au Canada }{ }^{14}\end{array}$ \\
\hline AHA-P & American Heart Association: Management of stroke in infants and children ${ }^{8}$ \\
\hline ASA & American Stroke Association ${ }^{3-7}$ \\
\hline $\mathrm{AU}$ & National Stroke Foundation, Australia: Clinical guidelines for acute stroke management ${ }^{11}$ \\
\hline AU-R & National Stroke Foundation, Australia: Clinical guidelines for stroke rehabilitation and recovery ${ }^{12}$ \\
\hline AVERT & A Very Early Rehabilitation Trial for stroke $e^{40,41}$ \\
\hline CAST/IST & Chinese Acute Stroke Trial/International Stroke Trial ${ }^{42}$ \\
\hline CCCDTD & Conférence canadienne de consensus sur le diagnostic et le traitement de la démence ${ }^{13}$ \\
\hline $\mathrm{CCF}$ & Canadian Continence Foundation ${ }^{43}$ \\
\hline CHARISMA & Clopidogrel for High Atherothrombotic Risk and Ischemic Stabilization, Management, and Avoidance ${ }^{44}$ \\
\hline Cochrane & Base de données des revues systématiques Cochrane : Thrombolysis for acute ischaemic stroke ${ }^{45}$ \\
\hline CSQCS & Canadian Stroke Quality of Care Study ${ }^{17-20}$ \\
\hline EBRSR & Evidence-Based Review of Stroke Rehabilitation ${ }^{24}$ \\
\hline ECASS III & European Cooperative Acute Stroke Study III: Thrombolysis with alteplase 3 to 4.5 hours after acute ischemic stroke ${ }^{46}$ \\
\hline ESO & European Stroke Organization: Guidelines for the management of ischaemic stroke and transient ischaemic attack ${ }^{25}$ \\
\hline EXPRESS & Early use of Existing Preventive Strategies for Stroke ${ }^{47}$ \\
\hline $\begin{array}{l}\text { Groupe } \\
\text { d'Ottawa }\end{array}$ & Evidence-based clinical practice guidelines for post-stroke rehabilitation ${ }^{23}$ \\
\hline HSFO & Heart and Stroke Foundation of Ontario: Consensus Panel on the Stroke Rehabilitation System ${ }^{48}$ \\
\hline MATCH & Management of Atherothrombosis with Clopidogrel in High-risk patients with recent TIA or ischemic stroke ${ }^{49}$ \\
\hline NAEMSP & National Association of EMS Physicians ${ }^{50}$ \\
\hline NICE & National Institute for Health and Clinical Excellence ${ }^{31}$ \\
\hline NOCP & Association des Paramédics du Canada : Profils des compétences du personnel paramédical ${ }^{51}$ \\
\hline NZ & Stroke Foundation, New Zealand: New Zealand guideline for management of stroke ${ }^{26}$ \\
\hline OCCPG & Lignes directrices canadiennes sur la prise en charge et la prévention de l'obésité s2 $^{2}$ \\
\hline $\mathrm{PECH}$ & Programme éducatif canadien sur l'hypertension ${ }^{16}$ \\
\hline PROGRESS & PROGRESS Collaborative Group: Randomised trial of a perindopril-based blood-pressure-lowering regimen ${ }^{53}$ \\
\hline $\mathrm{RCP}$ & Royal College of Physicians: National clinical guidelines for stroke $\mathrm{e}^{35}$ \\
\hline RCP-P & Royal College of Physicians: Stroke in childhood ${ }^{37}$ \\
\hline RNAO & Registered Nurses Association of Ontario: Nurses best practice guidelines (continence) ${ }^{22}$ \\
\hline SCORE & Stroke Canada Optimization of Rehabilitation through Evidence ${ }^{38}$ \\
\hline SIGN & Scottish Intercollegiate Guidelines Network: Management of patients with stroke ${ }^{27-30}$ \\
\hline SIGN 13 & $\begin{array}{l}\text { Scottish Intercollegiate Guidelines Network: Management of patients with stroke I: assessment, investigation, } \\
\text { immediate management and secondary prevention }{ }^{27}\end{array}$ \\
\hline SIGN 14 & $\begin{array}{l}\text { Scottish Intercollegiate Guidelines Network: Management of patients with stroke II: management of carotid } \\
\text { stenosis and carotid endarterectomy }\end{array}$ \\
\hline SIGN 64 & $\begin{array}{l}\text { Scottish Intercollegiate Guidelines Network: Management of patients with stroke IV: rehabilitation, prevention } \\
\text { and management of complications and discharge planning }\end{array}$ \\
\hline SIGN 78 & $\begin{array}{l}\text { Scottish Intercollegiate Guidelines Network: Management of patients with stroke III: identification and } \\
\text { management of dysphagia }{ }^{29}\end{array}$ \\
\hline VA/DoD & $\begin{array}{l}\text { US Veterans Affairs/Department of Defense: Clinical practice guideline for the management of stroke } \\
\text { rehabilitation }{ }^{10}\end{array}$ \\
\hline
\end{tabular}

*Dans bien des cas, les recommandations pour les pratiques optimales ont été adaptées de lignes directrices existantes ou concordent avec des lignes directrices existantes. Ces lignes directrices sont identifiées à la fin de chaque énoncé de recommandation, le cas échéant, au moyen d'abréviations citées dans la présente liste. 
auteurs ont entrepris une vaste consultation nationale afin de recueillir les commentaires judicieux des intervenants présents de première ligne et directement chargés d'appliquer les recommandations, de même que des responsables des systèmes de santé, des autorités sanitaires régionales et du gouvernement. La Stratégie canadienne de l'AVC et les groupes de travail ont pris connaissance de tous les commentaires et de toutes les suggestions pour la mise à jour de 2008. Le comité consensuel a également fourni des lignes directrices et formulé des commentaires. En dernier lieu, après l'application des changements suggérés, le comité consensuel est passé au vote et s'en est suivie une entente quasi-unanime sur les modifications suivantes.

\section{Révisions des recommandations actuelles pour les pratiques optimales}

- Le comité a proposé et approuvé des modifications et corrections mineures pour 21 des 24 recommandations originales pour les pratiques optimales.

- Les auteurs ont fondu en une seule recommandation relative à l'imagerie neurovasculaire les recommandations sur la tomodensitométrie (TDM) et l'imagerie carotidienne.

- Ils ont révisé en profondeur la recommandation sur la thrombolyse en phase aiguë, à la lumière des preuves récentes.

- Ils ont recentré la recommandation sur la réadaptation en milieu communautaire de façon à inclure des services de réadaptation ambulatoires et communautaires.

\section{Autres amendements}

- Planification du congé : La planification du congé doit débuter peu après que le patient se soit présenté dans un établissement de soins de santé, puis être révisée et mise à jour au besoin à chaque étape. Pour la version 2008, les auteurs ont apporté des modifications à certaines des recommandations existantes, selon le cas, de manière à rappeler l'importance de la planification du congé.

- AVC chez l'enfant : L'AVC peut survenir à tout âge. Bien qu'il soit plus rare chez les enfants, il risque d'entraîner de graves problèmes à long terme pour le patient qui y survit. Bon nombre des recommandations de ce document s'appliquent aux sujets de tous âges et tout au long du continuum des soins de l'AVC. Les Recommandations canadiennes pour les pratiques optimales de soins de l'AVC ne se veulent pas des lignes directrices complètes pour la prise en charge des enfants victimes d'un AVC. Des ajouts fondés sur des preuves ont plutôt été apportés pour souligner certaines problématiques particulières aux soins de l'AVC chez l'enfant. On trouve à la fin de ce document les références de lignes directrices détaillées et à jour sur l'AVC chez l'enfant ${ }^{8,37}$.

\section{Approbation de quatre nouvelles recommandations}

Le comité consensuel a approuvé quatre recommandations sur les sujets suivants :

- services médicaux d'urgence pour les patients victimes d'AVC avant leur arrivée à l'hôpital et durant leur transfert inter-hospitalier;
- gestion de la phase aiguë de l'accident ischémique transitoire et de l'AVC mineur, surtout chez les patients pris en charge dans la communauté ou qui retournent à la maison au sortir de l'urgence;

- éléments de la prise en charge de l'AVC en phase aiguë visant à réduire le risque de complications;

- atteinte cognitive et démence d'origine vasculaire comme manifestations de l'AVC, afin de rappeler que les symptômes d'une atteinte cognitive vasculaire sont une indication de la nécessité d'administrer un traitement de prévention secondaire agressif.

\section{Reconnaissance des obstacles à l'application des recommandations et leurs solutions}

Lors de sa réunion, le comité consensuel pour les recommandations 2008 a consacré énormément de temps à débattre des obstacles à l'application des Recommandations pour les pratiques optimales et à leurs solutions. Le comité s'est subdivisé en quatre groupes, soit soins en phase aiguë, prévention, réadaptation et rétablissement, tandis que le quatrième groupe, plus nombreux, a été chargé des questions relatives au système de santé.

Voici quelques-uns des facteurs nécessaires pour assurer une application fructueuse des recommandations :

- coordonnateurs des soins de l'AVC disposant de ressources suffisantes et engagés pour gérer l'application des recommandations;

- soutien et financement des gouvernements;

- découverte et participation de personnes influentes du milieu de l'AVC;

- intégration des programmes et des services pour l'AVC dans les plans stratégiques et opérationnels régionaux et hospitaliers;

- démonstration de l'impact économique de la prestation de soins coordonnés pour l'AVC et de l'application des Recommandations pour les pratiques optimales.

Les principaux obstacles à l'application des pratiques optimales de soins de l'AVC recensés sont : (1) la discordance entre les priorités établies au sein des systèmes de soins de santé, des régions et des établissements et (2) le manque de ressources humaines, financières et matérielles. Les auteurs ont noté ces facteurs à la rubrique «Exigences pour le système» de plusieurs recommandations, selon le cas.

\section{Ordre de priorité pour l'application des recommandations}

Dans le cadre de la réunion du comité consensuel 2008, les membres ont eu pour tâche finale de participer à un exercice de priorisation des recommandations en vue de leur application, dans le but d'aider à l'assignation des maigres ressources locales, régionales et nationales au chapitre des soins de l'AVC. Le groupe a toutefois jugé à l'unanimité que pour prodiguer des soins efficaces à tous les Canadiens victimes d'AVC, il faut des systèmes de soins coordonnés et intégrés, ce qui exigera l'application intégrale de toutes les recommandations pour les pratiques optimales contenues dans le présent document.

À la fin de la séance en petits groupes tenue en après-midi, on a posé la question suivante à tous les participants du comité 
consensuel : «En gardant à l'esprit que toutes les lignes directrices proposées dans les Recommandations canadiennes pour les pratiques optimales de soins de l'AVC (2008) sont importantes et nécessaires, lesquelles, si elles étaient appliquées immédiatement, auraient le plus grand impact sur les soins de l'AVC au Canada?». Voici une liste des dix priorités (selon leur importance en ordre décroissant) d'après le nombre le plus élevé de votes accordés par les membres du comité :

1. Prise en charge de l'accident ischémique transitoire et de l'AVC mineur

2. Réadaptation ambulatoire et en milieu communautaire

3. Mise sur pied d'unités d'AVC

4. Prise en charge de l'AVC par les services médicaux d'urgence

5. Évaluation initiale en vue de la réadaptation

6. Contrôle de la tension artérielle

7. Prestation des services de réadaptation pour les patients hospitalisés

8. Traitement de la dépression post-AVC

9. Interventions sur l'artère carotide

10. Traitement antithrombotique pour la fibrillation auriculaire

\section{Diffusion et mise en œuvre}

\section{Réseautage}

Plusieurs stratégies de diffusion des recommandations sur les pratiques optimales ont été définies et mises en œuvre à la suite de leur publication initiale en 2006. On continue de poursuivre bon nombre de ces stratégies.

- Consultation d'experts de la recherche dans le domaine de l'application des connaissances et de la mise en œuvre des recommandations au Canada et à l'étranger afin de définir et d'utiliser des stratégies d'application fondées sur des données probantes.

- Communication des progrès réalisés à tous les groupes de travail de la Stratégie canadienne de l'AVC afin d'assurer l'harmonisation et la collaboration dans la diffusion.

- Présentation aux champions provinciaux de l'AVC et discussion avec eux au cours des stades de la rédaction, de la création et de la production du contenu final.

- Consultation d'autres groupes nationaux de production de lignes directrices dans des domaines connexes (p. ex., hypertension, dyslipidémie, diabète).

- Présentation pour discussion au cours de réunions de professionnels de la santé dans toutes les disciplines des soins de santé et dans tout le continuum des soins de l'AVC aux échelons national, provincial et régional.

- Présentation à des professionnels de la santé de première ligne à l'échelon local et utilisation de mécanismes consensuels locaux pour revoir les facteurs habilitants et inhibiteurs de la mise en œuvre des recommandations, ainsi que des stratégies innovatrices de mise en œuvre, et pour en produire une évaluation structurée.

- Affichage des recommandations sur le site web de la Stratégie canadienne de l'AVC, ainsi que sur d'autres sites web qui constituent des dépôts centraux de lignes directrices.

- Envoi postal direct à des intervenants clés et à des professionnels de la santé de première ligne qui s'occupent des personnes qui ont subi un AVC et les membres de leur famille dans tout le continuum des soins.

- Points saillants de recommandations en particulier publiées dans des bulletins portant sur l'AVC comme celui du Conseil national des soins infirmiers de l'AVC.

- Mécanisme structuré de formulation de commentaires inclus dans des envois postaux et sur le site web de la Stratégie canadienne de l'AVC.

\section{Outils pour appuyer la mise en œuvre des recommandations sur les pratiques optimales}

Le programme national de formation et de perfectionnement professionnel de la Stratégie canadienne de l'AVC porte avant tout sur la mise en œuvre d'un plan de formation et de perfectionnement professionnel à l'intention des professionnels de la santé qui s'occupent de patients victimes d'un AVC. Le Groupe de travail sur la formation et le perfectionnement professionnel a établi une approche à trois volets qui englobe l'éducation préprofessionnelle, le perfectionnement professionnel et le changement de systèmes. Ce groupe de travail a effectué une évaluation nationale des besoins et déterminé qu'il fallait des outils au point de service pour faciliter l'application des recommandations sur les pratiques optimales relatives à l'AVC en contexte clinique.

Le Groupe de travail sur la formation et le perfectionnement professionnel a créé plusieurs outils au point de service qui sont maintenant disponibles sur les sites web de la Stratégie canadienne de l'AVC et de la Fondation des maladies du cœur du Canada.

- Ressources pour la prise en charge de l'AVC aigu

- Recommandations canadiennes pour les pratiques optimales de soins de l'AVC — Trousse d'outils de 2006

- Fiches de référence format poche : Nerfs crâniens, Manifestations courantes au cours de l'AVC, Fonctions du cerveau, Échelle de l'accident vasculaire cérébral des National Institutes of Health, Échelle neurologique canadienne, Fiche de prévention de l'AVC

- FAQ FAAST pour infirmières

- Atlas des ressources nationales en matière d'AVC

Les ressources de formation et de perfectionnement professionnel sur l'AVC demeureront un élément important de la stratégie de mise en application des Recommandations canadiennes pour les pratiques optimales de soins de l'AVC. La Fondation des maladies du cœur pilote les travaux en cours d'établissement des priorités et de production de ressources de perfectionnement professionnel en partenariat avec la Stratégie canadienne de l'AVC.

Sites web offrant de l'information sur le perfectionnement professionnel :

- Stratégie canadienne de l'AVC : www.canadianstrokestrategy.ca

- Site de formation professionnelle de la Fondation des maladies du cœur : http://profed.heartandstroke.ca

\section{Utilisation de ce document}

Les pages qui suivent présentent la version intégrale de cha- 
cune des recommandation, et les niveaux de preuve (A, B et C) sont définis au Tableau 2 de ce document abrégé. La justification, les indicateurs de rendement, la portée pour le système et les résumés des données probantes figurent dans la version intégrale des Recommandations canadiennes pour les pratiques optimales de soins de l'AVC, que l'on trouvera à www.cmaj.ca/content/vol179/issue12/\#supplement, accompagnés de renseignements supplémentaires au sujet du mécanisme d'élaboration et de mise à jour des lignes directrices de la Stratégie canadienne de l'AVC.

\section{1: Sensibilisation du public et enseignement au patient}

\section{Recommandation 1.1 : Sensibilisation et faculté de réponse du public}

Tout le monde devrait être en mesure de reconnaître les signes avant-coureurs et les symptômes de l'AVC qui comprennent une faiblesse, un trouble de la parole, un trouble de vision, un mal de tête et des étourdissements, apparaissant tous soudainement (voir l'Encadré 1).

i. Dans l'information transmise au public sur l'AVC, il faudrait insister sur le fait qu'il s'agit d'une urgence médicale et qu'il faut chercher à obtenir les services d'un médecin sur-le-champ. Tous les membres du public devraient connaître les mesures appropriées à prendre, c.-à-d. composer le 9-1-1 ou le numéro du service d'urgence local [niveau de preuve B] (CSQCS, ESO).

ii. La sensibilisation du public devrait inclure de l'information indiquant que l'AVC peut frapper à n'importe quel âge, du nouveau-né à l'adulte, en passant par les enfants [niveau de preuve C] (RCP-P).

\section{Recommandation 1.2 : Enseignement au patient et à sa famille}

Remarque : L'enseignement au patient, à sa famille et aux soignants fait partie intégrante des soins, à tous les stades $d u$ continuum des soins de l'AVC, tant chez l'adulte que chez l'enfant. Cet enseignement repose sur le transfert de connaissances théoriques et pratiques et peut inclure d'autres éléments de formation au besoin, notamment l'enseignement de techniques aux patients et à leur famille, pour l'auto-prise en charge ou pour la prise en charge des patients adultes et des enfants victimes d'AVC.
Un enseignement intégré et coordonné doit être fourni sans délai à toutes les étapes du continuum des soins de l'AVC, à tous les patients victimes d' $\mathrm{AVC}$ ou exposés à un risque d'AVC, de même qu'à leurs proches et à leurs soignants.

i. Le contenu didactique doit être adapté à la phase des soins ou de la récupération tout au long du continuum des soins de l'AVC ainsi qu'au patient, à sa famille et aux soignants, selon leur degré de préparation et leurs besoins [niveau de preuve $\mathrm{B}]^{55}$.

ii. La portée du contenu didactique doit couvrir tous les aspects des soins et du rétablissement, y compris la nature de l'AVC et ses manifestations, signes et symptômes, les atteintes, leur impact et leur prise en charge liée à la formation des soignants, les facteurs de risque, la dépression post-AVC, l'atteinte cognitive, la planification du congé et les processus décisionnels, les ressources, services et programmes de soutien communautaires et les modifications et avantages du milieu de vie [niveau de preuve A] (AU, CSQCS, Hare et coll. ${ }^{56}, \mathrm{NZ}, \mathrm{RCP}$ ).

iii. L'enseignement sera préférablement interactif, opportun, à jour, offert en diverses langues et sous diverses formes (document écrit, communication orale, accessible aux patients aphasiques ou par approche de thérapie de groupe) et propre aux besoins et aux atteintes des patients, ainsi qu'aux besoins de leurs proches et de leurs soignants. La prestation de l'enseignement doit veiller à

\section{Encadré 1 : Signes annonciateurs de l'AVC*.}

- Faiblesse : faiblesse subite, engourdissement ou picotement au visage, aux bras ou aux jambes

- Troubles du langage : incapacité subite et transitoire de parler ou de comprendre le langage

- Troubles visuels : perte subite de vision, particulièrement d'un œil, ou vision double

- Céphalées : céphalées subites, intenses et inhabituelles

- Étourdissements : perte subite d'équilibre, surtout accompagnée de l'un ou l'autre des signes mentionnés plus haut

Mesure à prendre : composer immédiatement le 9-1-1 ou le numéro de l'urgence de votre localité.

* D'après Fondation des maladies du cœur du Canada : www.heartandstroke.ca

Tableau 2 : Sommaire des définitions des niveaux de preuve utilisés dans ce document ${ }^{54}$.

\begin{tabular}{ll}
\hline Catégorie & Critères \\
\hline A & $\begin{array}{l}\text { Recommandation solide. Preuves tirées d'essais contrôlés et randomisés ou de méta-analyses d'essais contrôlés et } \\
\text { randomisés. Les effets souhaités surpassent nettement les effets indésirables ou vice versa. }\end{array}$ \\
B & $\begin{array}{l}\text { Essai contrôlé et randomisé simple ou étude d'observation bien conçue, donnant des preuves solides; étude de } \\
\text { cohorte ou étude analytique avec cas-témoins bien conçues; séries chronologiques multiples; résultats } \\
\text { déterminants d'une expérience non contrôlée. Les effets souhaités et les effets indésirables s'équivalent. }\end{array}$ \\
C & $\begin{array}{l}\text { Au moins une étude descriptive non expérimentale bien conçue (p. ex., études comparatives, études de } \\
\text { corrélation, études de cas) ou rapports de comités d'experts, opinions et(ou) expériences de sommités, y compris } \\
\text { consensus au sein de groupes de rédacteurs et(ou) d'examinateurs. }\end{array}$ \\
\hline
\end{tabular}


ce que les survivants d'un AVC puissent y accéder [niveau de preuve B] (AU, CSQCS, NZ, RCP).

iv. Les médecins et(ou) les équipes doivent élaborer des procédés pour un enseignement périodique aux patients, aux soignants et aux proches, au cours duquel des membres désignés de l'équipe seront responsables de transmettre des notions, documentations à l'appui [niveau de preuve C] (ASA).

\section{2 : Prévention de l'AVC (voir l'Encadré 2)}

\section{Recommandation 2.1 : Gestion du mode de vie et des facteurs de risque}

Chez les individus à risque d'AVC et chez ceux qui en ont subi un, il faut rechercher les facteurs de risque de maladie vasculaire et réviser le mode de vie (alimentation, apport en sodium, exercice, poids, tabagisme et consommation d'alcool). Les personnes concernées devraient être informées des stratégies possibles pour modifier leurs facteurs de risque et leur mode de vie [niveau de preuve B] (AU, NZ, RCP, $\mathrm{VA} / \mathrm{DoD})$

Les interventions portant sur les modes de vie et les facteurs de risque doivent inclure les suivantes :

i. Alimentation saine et équilibrée : Conformément au Guide alimentaire canadien pour manger sainement [niveau de preuve B] (ASA, PECH, RCP) une alimentation saine et équilibrée doit être riche en fruits et légumes frais, produits laitiers faibles en gras, fibres alimentaires et solubles, grains entiers et protéines d'origine végétale, et doit être faible en gras saturés, cholestérol et sodium.

ii. Sodium : L'apport recommandé de sodium par jour provenant de toutes sources équivaut à l'Apport suffisant selon l'âge. Pour les personnes de 9 à 50 ans, l'Apport suffisant est de $1500 \mathrm{mg}$, tandis qu'il passe à $1300 \mathrm{mg}$ pour les personnes de 50 à 70 ans et à $1200 \mathrm{mg}$ pour les personnes de plus de 70 ans. Aucun groupe d'âge ne devrait dépasser un apport maximal quotidien de sodium de $2300 \mathrm{mg}$ [niveau de preuve B].

Encadré 2 : Définitions de la prévention dans le contexte des Recommandations canadiennes pour les pratiques optimales de soins de l'AVC.

- Prévention primaire : La prévention primaire est une approche clinique à la prévention de la maladie qui est établie au cas par cas. Elle est axée sur la prévention d'une première occurrence de la maladie chez des sujets par ailleurs en bonne santé ${ }^{57,58}$. On applique généralement la prévention primaire dans un contexte de soins primaires; le médecin, l'infirmière en pratique avancée ou le patient peuvent amorcer la discussion sur la réduction du risque d'AVC. La prévention primaire et les recommandations relatives à la promotion de la santé en lien avec l'AVC (mode de vie et correction des facteurs de risque, dépistage de I'hypertension et des dyslipidémies et prise en charge du diabète) soulignent l'importance du dépistage et de la surveillance des patients exposés à un risque élevé de premier AVC. La prévention primaire et la réduction de la prévalence des facteurs de risque dans la population générale ne sont pas les principaux buts visés par les recommandations actuelles pour les pratiques optimales de soins de l'AVC; par conséquent, seules des recommandations sélectionnées ayant trait à la prévention primaire s'y retrouvent. Les auteurs travaillent actuellement à rédiger un ensemble complet de recommandations à cet égard. Elles seront incluses dans les mises à jour ultérieures.

- Prévention secondaire : La prévention secondaire de l'AVC est une approche clinique qui est établie au cas par cas dans le but de réduire le risque de récurrence d'accidents vasculaires chez les personnes qui ont déjà subi un AVC ou un accident ischémique transitoire et chez les sujets qui présentent un ou plusieurs problèmes de santé ou facteurs de risque qui les exposent à un «risque élevé d'AVC» ${ }^{58}$. Les recommandations en matière de prévention secondaire contenues dans le présent document concernent les facteurs de risque les plus pertinents dans le contexte de I'AVC, soit notamment le mode de vie (alimentation, apport en sodium, tabagisme, exercice, poids et consommation d'alcool), I'hypertension, la dyslipidémie, les antécédents d'AVC ou d'accident ischémique transitoire, la fibrillation auriculaire et l'AVC et la sténose carotidienne.

- Les recommandations en matière de prévention secondaire présentées dans cette partie du document peuvent servir dans divers contextes, tels que les soins actifs, les cliniques de prévention de l'AVC et les soins communautaires. Elles s'adressent aux patients qui ont d'abord été vus en soins primaires, à ceux qui sont vus dans un service d'urgence et reçoivent leur congé et à ceux qui sont hospitalisés en raison d'un AVC ou d'un accident ischémique transitoire. II faut appliquer les recommandations en matière de prévention secondaire de l'AVC tout au long de la phase de rétablissement, y compris durant la réadaptation à l'hôpital ou en clinique externe, la réintégration dans la communauté et le suivi permanent effectué par les médecins de soins primaires. Après un AVC ou un accident ischémique transitoire, il faudra aborder les questions de prévention secondaire à chaque consultation avec un professionnel de la santé.

- Veuillez aussi consulter la recommandation 3.2, «Prise en charge active de l'accident ischémique transitoire et de I'AVC mineur» pour d'autres indications sur l'évaluation du risque d'AVC. 
Voir le site www.sodium101.ca pour des lignes directrices sur l'apport en sodium.

iii. Exercice : Exercice modéré : marche (idéalement marche rapide), jogging, bicyclette, natation ou autre exercice dynamique totalisant 30 à 60 minutes/jour à raison de quatre à sept jours par semaine en plus des activités routinières de la vie quotidienne [niveau de preuve A]. On recommande des programmes d'exercice surveillés par un médecin chez les patients à risque élevé (p. ex., ceux qui ont une cardiopathie) (ASA, PECH, EBRSR, NZ).

iv. Poids : Maintenir un indice de masse corporelle (IMC) de 18,5 à $24,9 \mathrm{~kg} / \mathrm{m}^{2}$ et un tour de taille de $<88 \mathrm{~cm}$ chez la femme et de $<102 \mathrm{~cm}$ chez l'homme [niveau de preuve B] (ASA, PECH, OCCPG).

v. Tabagisme : Abandon du tabac et environnement sans fumée; thérapie de remplacement de la nicotine et thérapie comportementale [niveau de preuve B] (ASA, $\mathrm{PECH}, \mathrm{CSQCS}, \mathrm{RCP})$. Comme thérapie de remplacement de la nicotine, il faudrait envisager la nortriptyline, des agonistes partiels des récepteurs de la nicotine ou une thérapie comportementale [niveau de preuve A] (ASA, AU).

vi. Consommation d'alcool : Deux consommations ordinaires ou moins par jour et moins de 14 consommations par semaine chez l'homme, et moins de neuf chez la femme [niveau de preuve C] (ASA, AU, PECH).

\section{Recommandation 2.2 : Gestion de la tension artérielle}

L'hypertension est le facteur de risque modifiable le plus important à l'égard de l'AVC. Il faut vérifier la tension artérielle (TA) chez toutes les personnes à risque d'un AVC.

\section{2a. Mesure de la tension artérielle}

i. Il faut mesurer la tension artérielle de toutes les personnes exposées à un risque d'AVC à chaque consultation avec un professionnel de la santé, mais pas moins d'une fois l'an [niveau de preuve C] (PECH, NICE, RCP).

ii. Il faut appliquer les techniques appropriées normalisées préconisées par le Programme éducatif canadien sur l'hypertension $(\mathrm{PECH})$ pour la mesure de la tension artérielle.

iii. Il faut soumettre les patients dont la tension artérielle est élevée à un examen approfondi pour diagnostic d'hypertension, conformément aux directives du Programme éducatif canadien sur l'hypertension $(\mathrm{PECH})$ [niveau de preuve A] (ASA, PECH, RCP).

iv. Il faut conseiller un mode de vie sain aux patients hypertendus ou exposés à un risque d'hypertension [niveau de preuve C]. Consultez la recommandation 2.1, «Gestion du mode de vie et des facteurs de risque», pour plus de détails sur les modifications à apporter au mode de vie.

\section{2b. Contrôle de la tension artérielle}

i. La Stratégie canadienne de l'AVC recommande des valeurs cibles de tension artérielle, telles qu'elles sont définies dans les directives du Programme éducatif canadien sur l'hypertension (PECH) pour la prévention d'un premier $\mathrm{AVC}$, des récidives d'AVC et autres événements vasculaires.

Recommandations du PECH 2008 pour la prise en charge de la tension artérielle (Extraits utilisés avec permission. Consulter www.hypertension.ca/chep ${ }^{16}$.) (Adaptation libre)

- Pour la prévention d'un premier AVC dans la population générale, l'objectif thérapeutique pour le traitement de l'hypertension artérielle systolique est une TA inférieure à $140 \mathrm{~mm} \mathrm{Hg}$ [niveau de preuve C]. L'objectif thérapeutique pour le traitement de l'hypertension artérielle diastolique est une TA inférieure à $90 \mathrm{~mm} \mathrm{Hg}$ [niveau de preuve A].

- Un traitement antihypertenseur est recommandé chez tout patient qui a été victime d'un AVC ou d'un accident ischémique transitoire, ciblant une TA inférieure à 140/90 mm Hg [niveau de preuve C].

- Chez les patients qui ont subi un AVC, on préférera un traitement par inhibiteur de l'enzyme de conversion de l'angiotensine (IECA) ou par diurétique [niveau de preuve B].

- Un traitement antihypertenseur est recommandé pour la prévention d'un premier AVC ou des récidives d'AVC chez les patients diabétiques, de manière à atteindre une TA systolique inférieure à $130 \mathrm{~mm} \mathrm{Hg}$ [niveau de preuve $\mathrm{C}$ ] et une $\mathrm{TA}$ diastolique inférieure à $80 \mathrm{~mm} \mathrm{Hg}$ [niveau de preuve A].

- Un traitement antihypertenseur est recommandé pour la prévention d'un premier AVC ou des récidives d'AVC chez les patients non diabétiques souffrant d'insuffisance rénale chronique, de manière à atteindre une TA inférieure à 130/80 mm Hg [niveau de preuve C] .

ii. Aucun essai contrôlé n'a encore déterminé le moment idéal pour instaurer le traitement antihypertenseur après l'AVC ou l'accident ischémique transitoire. On recommande de commencer (ou de modifier) le traitement antihypertenseur avant le congé de l'hôpital. Chez les patients victimes d'un AVC non invalidant ou d'un accident ischémique transitoire, ne nécessitant pas d'hospitalisation, on recommande de commencer (ou de modifier) le traitement antihypertenseur au moment du premier examen médical [niveau de preuve B] (EXPRESS, PROGRESS).

iii. Pour des recommandations sur des agents ou séquences d'agents spécifiques, veuillez consulter les lignes directrices actuelles du PECH (Programme éducatif canadien sur l'hypertension) ${ }^{16}$.

\section{Recommandation 2.3 : Gestion des lipides}

Les taux de lipides devraient être surveillés chez les personnes à risque d'AVC.

\section{3a. Évaluation des lipides}

i. Le taux sérique des lipides à jeun [cholestérol total, triglycérides totaux, cholestérol LDL (lipoprotéine de basse densité) et cholestérol HDL (lipoprotéine de haute 
densité)] devrait être mesuré tous les 1 à 3 ans chez tous les hommes de 40 ans et plus et chez toutes les femmes postménopausées ou qui ont 50 ans et plus [niveau de preuve C] (McPherson et al. ${ }^{15}$, VA/DoD). On recommande de faire des dosages plus fréquents pour les patients ayant des taux anormaux ou si un traitement est amorcé.

ii. Quel que soit l'âge, les adultes présentant un diabète, un tabagisme, une hypertension, une obésité, une cardiopathie ischémique, des troubles rénovasculaires, des troubles vasculaires périphériques, une sténose carotidienne asymptomatique ou des antécédents d'accident ischémique cérébral ou d'accident ischémique transitoire devraient faire l'objet d'un dépistage [niveau de preuve C] (McPherson et al. ${ }^{15}$ ).

\section{3b. Gestion des lipides}

i. Il faudrait prendre en charge les patients ayant eu un AVC ischémique dont le taux de cholestérol LDL est de $>$ 2,0 $\mathrm{mmol} / \mathrm{L}$ au moyen d'un programme de modification du mode de vie et de lignes directrices alimentaires [niveau de preuve A] (AU, CSQCS, McPherson et coll. $\left..^{15}, \mathrm{VA} / \mathrm{DoD}\right)$.

ii. Il faudrait prescrire des statines à la plupart des patients qui ont eu un AVC ischémique ou un accident ischémique transitoire pour atteindre un taux de lipides recommandé [niveau de preuve A] (AU, CSQCS, McPherson et coll. ${ }^{15}$, VA/DoD).

\section{Recommandation 2.4 : Prise en charge du diabète}

\section{4a. Évaluation du diabète}

i. Il faudrait, chaque année, évaluer le risque de diabète de type 2 chez toutes les personnes en fonction de critères démographiques et cliniques [niveau de preuve C] (ACD).

ii. Une mesure de la glycémie à jeun devrait être effectuée tous les 3 ans chez les personnes de plus de 40 ans pour dépister le diabète [niveau de preuve $\mathrm{C}$ ] $(\mathrm{ACD})$. Des dosages plus fréquents ou précoces de la glycémie à jeun ou 2 heures après l'absorption orale d'une charge de glucose de 75 g devraient être envisagés chez les personnes présentant d'autres facteurs de risque de diabète [niveau de preuve C] (ACD). Parmi ces facteurs, mentionnons les antécédents familiaux, une population à haut risque, une maladie vasculaire, des antécédents de diabète gestationnel, l'hypertension, la dyslipidémie, la surcharge pondérale, l'obésité abdominale et le syndrome des ovaires polykystiques.

iii. Les taux de lipides à jeun (cholestérol total, cholestérol HDL, triglycérides et cholestérol LDL calculé) devraient être évalués chez les adultes lorsqu'un diagnostic de diabète est posé, puis tous les 1 à 3 ans selon les indications cliniques. Le dosage des lipides devrait être effectué plus fréquemment si un traitement de la dyslipidémie est amorcé [niveau de preuve C] (ACD).

iv. La tension artérielle devrait être mesurée à chaque visite pour le diabète [niveau de preuve $\mathrm{C}](\mathrm{ACD})$.

\section{4b. Prise en charge du diabète}

i. Les cibles glycémiques doivent être personnalisées [niveau de preuve C] (ACD, ESO); toutefois, un traitement du diabète de type 1 ou de type 2 devrait viser un taux d'hémoglobine glyquée $\left(\mathrm{HB}_{\mathrm{Alc}}\right) \leq 7,0 \%$ pour réduire le risque de complications microvasculaires [niveau de preuve A] (ACD) et macrovasculaires [niveau de preuve C] (ACD).

ii. Pour atteindre un taux d'hémoglobine $\mathrm{HB}_{\mathrm{Alc}} \leq 7,0 \%$, les patients diabétiques de type 1 ou de type 2 devraient viser une glycémie à jeun ou préprandiale de 4,0 à 7,0 $\mathrm{mmol} / \mathrm{L}$ [niveau de preuve B] (ACD).

iii. La cible glycémique à 2 heures postprandiale est de 5,0 à $10,0 \mathrm{mmol} / \mathrm{L}$ [niveau de preuve B]. Si on ne peut atteindre les cibles de $\mathrm{HB}_{\mathrm{Alc}}$ en visant une glycémie postprandiale de 5,0 à $10,0 \mathrm{mmol} / \mathrm{L}$, on peut envisager de réduire davantage la glycémie, jusqu'à 5,0 à $8,0 \mathrm{mmol} / \mathrm{L}$ [niveau de preuve C] (ACD).

iv. Les adultes à haut risque d'épisode vasculaire devraient recevoir un traitement de statines pour obtenir un taux de cholestérol $\mathrm{LDL} \leq 2,0 \mathrm{mmol} / \mathrm{L}$ [niveau de preuve $\mathrm{A}$ ] (ACD).

v. Sauf si cela est contre-indiqué, un traitement à faible dose d'acide acétylsalicylique (AAS) (80 à 325 mg/jour) est recommandé pour tous les patients diabétiques qui souffrent de maladie cardiovasculaire ainsi que pour les personnes présentant des facteurs de risque d'athérosclérose indiquant une probabilité accrue d'événements cardiovasculaires [niveau de preuve $\mathrm{A}$ ] (ACD).

\section{Recommandation 2.5 : Traitement antiplaquettaire}

Il faudrait prescrire à tous les patients qui ont subi un accident ischémique cérébral ou un accident ischémique transitoire un traitement antiplaquettaire pour la prévention secondaire des récidives d'AVC à moins qu'une anticoagulation soit indiquée [niveau de preuve A] (ASA, AU, CSQCS, ESO, NZ, RCP, VA/DoD).

i. On peut utiliser l'AAS seul, l'association AAS (25 mg) et dipyridamole à libération prolongée (200 mg), ou le clopidogrel, selon les circonstances cliniques [niveau de preuve A].

ii. Chez les patients adultes qui prennent de l'AAS, la posologie d'entretien habituelle est de 80 à $325 \mathrm{mg}$ par jour [niveau de preuve A] (CSQCS, VA/DoD) et chez les enfants victimes d'un AVC, la posologie d'entretien habituelle à l'AAS est de 3 à $5 \mathrm{mg} / \mathrm{kg}$ par jour pour prévenir les récidives d'AVC [niveau de preuve C] (AHA-P).

iii. On ne recommande pas les combinaisons AAS et clopidogrel de longue durée pour la prévention des AVC secondaires [niveau de preuve B] (CHARISMA, MATCH).

\section{Recommandation 2.6 : Traitement antithrombotique pour la fibrillation auriculaire}

Les patients ayant eu un AVC et présentant une fibrillation auriculaire devraient recevoir de la warfarine, visant un rapport international normalisé (RIN) de 2,5 - intervalle thérapeutique de 2,0 à 3,0 (RIN cible de 3,0 dans le cas de 
valves cardiaques mécaniques - intervalle thérapeutique de 2,5 à 3,5) [niveau de preuve A], s'ils sont susceptibles de respecter la surveillance nécessaire et ne sont pas à haut risque d'hémorragie (ASA, AU, CSQCS, ESO, SIGN, VA/DoD).

\section{Recommandation 2.7 : Intervention sur l'artère carotide}

\section{7a Sténose carotidienne symptomatique}

Il faudrait offrir aux patients ayant eu un accident ischémique transitoire ou un AVC non invalidant et présentant une sténose carotidienne interne ipsilatérale de 70 à $99 \%$ (mesurée par angiographie ou par deux techniques d'imagerie non invasives concordantes) une endartériectomie carotidienne dans les deux semaines qui suivent l'épisode d'AVC ou l'accident ischémique transitoire, sauf si cela est contreindiqué [niveau de preuve A] (ASA, AU, CSQCS, ESO, NZ, SIGN 14).

i. On recommande une endartériectomie carotidienne pour certains patients présentant une sténose symptomatique modérée (50 à 69\%). Ces patients devraient être évalués par un médecin spécialiste en AVC [niveau de preuve A] (ASA, AU, CSQCS, NZ, SIGN 14).

ii. L'endartériectomie carotidienne devrait être effectuée par un chirurgien dont le taux de morbidité et de mortalité périopératoire est connu et $<6 \%$ [niveau de preuve A] (ASA, CSQCS, ESO, NZ).

iii. L'installation d'un tuteur carotidien peut être envisagée chez les patients qui ne sont pas candidats à la chirurgie pour des raisons techniques, anatomiques ou médicales [niveau de preuve $\mathrm{C}$ ].

iv. L'endartériectomie carotidienne est contre-indiquée pour les patients atteints de sténose modérée $(<50 \%)$ [niveau de preuve A] (ASA, CSQCS, SIGN 14).

\section{7b Sténose carotidienne asymptomatique}

On pourrait envisager une endartériectomie carotidienne chez certains patients présentant une sténose carotidienne asymptomatique de 60 à $99 \%$.

i. Les patients doivent avoir moins de 75 ans et présenter un risque chirurgical de $<3 \%$, avoir une espérance de vie de $>5$ ans et être évalués par un médecin spécialiste en AVC [niveau de preuve A] (CSQCS, AU, AAN, AHA).

\section{3 : Gestion de l'AVC en phase hyperaiguë}

Cette section des recommandations décrit les soins de la phase hyperaiguë de l'AVC depuis le premier contact de la présumée victime d'un $\mathrm{AVC}$ avec l'équipe médicale jusqu'à son hospitalisation ou son congé de l'hôpital pour réintégrer la communauté.

\section{Recommandation 3.1 : Prise en charge des patients victimes d'AVC en phase aiguë dans les services médicaux d'urgence (nouvelle en 2008)}

Cette recommandation concerne la prise en charge des patients chez qui l'on soupçonne un AVC, depuis leur premier contact avec les services médicaux d'urgence locaux jusqu'à ce qu'ils soient confiés au personnel hospitalier, de même que celle des patients chez qui un AVC est soupçonné ou confirmé et qui sont transférés d'un établissement de soins de santé à un autre en utilisant les services médicaux d'urgence.

Cette recommandation s'adresse aux ambulanciers paramédicaux et aux personnes qui soutiennent les services médicaux d'urgence, notamment les responsables des communications et les répartiteurs. Elle concerne également les premiers intervenants (p. ex., les intervenants des unités médicales d'urgence et les paramédics de premier recours) qui ont reçu la formation appropriée pour faire le dépistage de l'AVC et prendre en charge les patients potentiellement victimes d'un AVC durant leur transfert.

Les patients qui manifestent des signes et des symptômes d'un AVC hyperaigu, habituellement défini par leur survenue au cours des 4,5 heures précédentes, doivent être traités comme des cas d'urgence chez qui chaque minute compte et transportés sans délai vers le plus proche établissement de santé capable de leur prodiguer des soins d'urgence pour l'AVC [niveau de preuve C] (ASA, AU, ESO, RCP).

i. On recommande fortement aux patients ou aux personnes présentes de communiquer immédiatement avec les services médicaux d'urgence (p. ex., 9-1-1) pour accélérer l'accès aux soins pour l'AVC aigu [niveau de preuve C] (ASA, ESO).

ii. Les répartiteurs des services médicaux d'urgence doivent considérer les patients qui manifestent des signes et symptômes d'AVC hyperaigu comme des cas prioritaires [niveau de preuve C] (ASA, AU, ESO, NAEMSP, RCP).

iii. Pour dépister l'AVC aigu, les ambulanciers (paramédics) doivent utiliser un outil diagnostique normalisé conformément au National Occupational Competency Profile $^{51}$ (NOCP) [niveau de preuve B] (ASA, AU, ESO).

iv. Il faut optimiser la prise en charge extrahospitalière du patient chez qui l'on soupçonne un AVC aigu pour répondre à ses besoins [niveau de preuve A] (ASA, $\mathrm{RCP})$.

v. Des protocoles de transport direct doivent être en place pour faciliter l'orientation des patients admissibles vers l'établissement le plus approprié et le plus proche capable de prodiguer les soins requis pour l'AVC aigu [niveau de preuve C] (AU, ESO).

vi. Le protocole de transport direct doit utiliser des critères basés sur : 1) un intervalle combinant la durée des symptômes et la durée anticipée du transport, le tout devant respecter la fenêtre thérapeutique, et(ou) 2) les besoins $\mathrm{du}$ patient en matière de soins aigus [niveau de preuve B] (ASA).

vii. Il faut tenter d'obtenir l'historique de l'événement du patient (s'il le peut) ou de son entourage, y compris les signes et symptômes et l'heure de leur apparition, de même que les antécédents médicaux et pharmaceutiques. [niveau de preuve C] (RCP). 
viii. Les ambulanciers (paramédics) doivent aviser l'établissement de destination de l'arrivée d'un patient soupçonné de souffrir d'un AVC aigu pour que l'équipe se prépare à l'accueillir [niveau de preuve C] (ASA, ESO, NAEMSP, RCP).

ix. Les ambulanciers (paramédics) doivent transférer le patient sans délai au personnel de l'établissement qui l'accueille. [niveau de preuve C].

\section{Recommandation 3.2 : Prise en charge aiguë de l'accident ischémique transitoire et de l'AVC mineur (nouvelle en 2008)}

Les patients qui présentent des symptômes évocateurs d'AVC mineur ou d'accident ischémique transitoire doivent faire l'objet d'une évaluation qui vise à confirmer le diagnostic et à commencer le traitement dès que la situation clinique le permet dans le but de réduire le risque d'AVC majeur.

\section{2a Évaluation}

i. Il faut soumettre tous les patients chez qui l'on soupçonne un accident ischémique transitoire ou un AVC mineur à un examen clinique immédiat et à d'autres épreuves diagnostiques au besoin, de manière à confirmer ou infirmer le diagnostic d'AVC et à élaborer un plan de soins [niveau de preuve B] (ASA, AU, CSQCS, ESO, EXPRESS, RCP).

ii. On recommande le recours à un outil de stratification du risque normalisé dès le premier contact avec les professionnels de la santé, qu'il s'agisse de soins primaires, secondaires ou tertiaires, afin de guider le processus de triage [niveau de preuve B] (AU, CSQCS).

iii. Diriger les patients chez qui l'on soupçonne un accident ischémique transitoire ou un AVC mineur vers une clinique de prévention de l'AVC désignée ou vers un médecin qui connaît bien l'évaluation et la prise en charge de l'AVC ou, en l'absence de telles options, vers un service d'urgence qui dispose d'un accès à un laboratoire d'imagerie neurovasculaire et à un personnel médical expérimenté en AVC [niveau de preuve B] (CSQCS, ESO, EXPRESS, SIGN 13).

iv. Les patients chez qui l'on soupçonne un accident ischémique transitoire ou un AVC mineur doivent subir des épreuves d'imagerie cérébrale par TDM ou imagerie par résonance magnétique (IRM). Les patients dont l'état est jugé très urgent (les patients les plus à risque d'une récidive d'AVC) doivent subir une épreuve d'imagerie neurovasculaire dans les 24 heures, et les patients dont l'état est jugé urgent doivent subir leur épreuve d'imagerie neurovasculaire dans les sept jours [niveau de preuve B] (ASA, AU, CSQCS, ESO, SIGN 13).

v. Les patients qui seraient des candidats à une revascularisation carotidienne doivent subir une angiotomographie, une angiographie par résonance magnétique ou une échographie carotidienne duplex le plus rapidement possible (dans les 24 heures pour les cas très urgents et dans les sept jours pour les cas urgents) [niveau de preuve C] (AU, CSQCS).

vi. Procéder d'emblée aux épreuves diagnostiques suivantes chez les patients chez qui l'on soupçonne un accident ischémique transitoire ou un AVC mineur : formule sanguine complète, électrolytes, fonction rénale, taux de cholestérol, glycémie et électrocardiographie [niveau de preuve C] (AU).

vii. Les patients chez qui l'on soupçonne un accident ischémique transitoire ou un AVC mineur et pour lesquels la présence d'un infarctus cérébral est confirmée à l'imagerie cérébrale doivent subir une évaluation ambulatoire complète visant à dépister une atteinte fonctionnelle, ce qui inclut une évaluation cognitive, un dépistage de la dépression, une vérification de l'aptitude à conduire, de même que des évaluations fonctionnelles en vue d'une éventuelle réadaptation [niveau de preuve B] (RCP), préférablement dans les deux semaines [niveau de preuve C]. Consulter la recommandation 5.1 «Évaluation initiale en vue de la réadaptation post-AVC» et la recommandation 5.5 «Suivi et réintégration dans la communauté» pour plus de détails.

\section{2b Prise en charge (Consulter la partie 2, "Prévention de l'AVC» pour plus de détails)}

i. Dès que l'imagerie cérébrale a exclu la présence d'une hémorragie intracrânienne, un traitement antiplaquettaire doit être administré chez tous les patients victimes d'un accident ischémique transitoire ou d'un AVC mineur qui ne prennent pas d'antiplaquettaires au moment de consulter [niveau de preuve A] (ASA, CAST/IST, ESO, RCP). La dose initiale d'AAS doit être d'au moins 160 $\mathrm{mg}$. La dose de charge de clopidogrel est de $300 \mathrm{mg}$. Consulter la recommandation 2.5 «Traitement antiplaquettaire» pour plus de détails sur le traitement antiplaquettaire à long terme.

ii. Pour les patients qui sont par ailleurs bons candidats à la revascularisation carotidienne, on préconise l'endartériectomie carotidienne le plus rapidement possible, soit dans les deux semaines de l'incident, chez les patients victimes d'un accident ischémique transitoire ou d'un AVC mineur avec sténose carotidienne supérieure à $70 \%$ et chez des patients sélectionnés atteints de sténose carotidienne symptomatique aiguë atteignant 50 à $69 \%$ du côté impliqué par leurs déficits neurologiques [niveau de preuve A] (AU, CSQCS, ESO, NICE, NZ, SIGN 14). Consulter la recommandation 2.7 «Intervention sur l'artère carotide» pour plus de détails.

iii. Les patients victimes d'un accident ischémique transitoire ou d'un AVC mineur et qui présentent une fibrillation auriculaire doivent recevoir un traitement anticoagulant par warfarine dès que l'imagerie cérébrale a exclu la présence d'une hémorragie intracrânienne; l'objectif thérapeutique est alors un rapport international normalisé de 2-3 [niveau de preuve A] (AU, CSQCS, ESO, NICE, NZ, SIGN 14). Consulter la recommandation 2.6 «Traitement antithrombotique pour la fibrillation auriculaire» pour plus de détails.

iv. Prendre en charge de manière énergique tous les facteurs de risque de maladie vasculaire au moyen d'interventions pharmacologiques et non pharmacologiques en 
visant une maîtrise optimale [niveau de preuve A] (ESO). S'il manque de preuves à l'appui des bienfaits que procure une modification des facteurs de risque individuels durant la phase aiguë, de telles preuves existent lorsque l'on applique une approche globale, par antihypertenseurs et statines, par exemple (Étude EXPRESS). Consulter la recommandation 2.2, «Contrôle de la tension artérielle» et 2.3, «Maîtrise des lipides» pour plus de détails.

v. Conseiller fortement aux patients victimes d'un accident ischémique transitoire ou d'un AVC mineur qui fument la cigarette de cesser immédiatement et appliquer les mesures pharmacologiques et non pharmacologiques qui s'imposent [niveau de preuve B] (ASA, CSQCS, ESO, $\mathrm{RCP})$.

\section{Recommandation 3.3 : Imagerie neurovasculaire}

Remarque : Cette recommandation sur l'imagerie neurovasculaire a été élaborée à partir de deux recommandations de l'édition 2006 des Recommandations canadiennes pour les pratiques optimales des soins de l'AVC : Imagerie du cerveau et imagerie carotidienne.

Il faut soumettre sans délai tous les patients chez qui l'on soupçonne un AVC aigu ou un accident ischémique transitoire à une épreuve d'imagerie cérébrale [niveau de preuve $\mathrm{A}$ ] (ASA, CSQCS).

i. Dans la plupart des cas, la modalité initiale choisie est une TDM sans agent de contraste [niveau de preuve B] (ASA, CSQCS).

ii. L'imagerie vasculaire doit être faite le plus tôt possible afin de mieux comprendre la cause de l'AVC et d'orienter les décisions thérapeutiques. L'imagerie vasculaire peut inclure une angiotomographie assistée par ordinateur, une angiographie par résonance magnétique, une angiographie par cathéter et une échographie duplex [niveau de preuve B] (ASA).

iii. Si l'on opte pour l'IRM, cette dernière doit inclure des séquences pondérées en diffusion afin de déceler l'ischémie, la séquence d'écho de gradient et la séquence d'inversion-récupération FLAIR (fluid-attenuated inversion recovery) afin de déterminer l'ampleur de l'infarctus ou la présence d'une hémorragie [niveau de preuve B] (CSQCS, NZ, RCP).

iv. Chez les enfants, si la TDM initiale est négative, il faut effectuer une IRM pour étayer le diagnostic et le plan de traitement [niveau de preuve B] (AHA-P).

v. L'imagerie carotidienne est indiquée dans les 24 heures suivant un accident ischémique transitoire dans le territoire irrigué par la carotide ou un AVC ischémique non invalidant (si l'épreuve n'a pas été effectuée lors de l'évaluation originale), à moins que le patient ne soit clairement pas un bon candidat pour l'endartériectomie carotidienne [niveau de preuve B] (CSQCS, SIGN 14).

vi. Chez les enfants, les artères cérébrales et cervicales doivent être examinées par imagerie le plus rapidement possible, préférablement dans les 24 heures [niveau de preuve C] (AHA-P).

\section{Recommandation 3.4 : Anomalies de la glycémie}

Il faut faire immédiatement le dosage de la glycémie de tous les patients chez qui l'on soupçonne un AVC aigu.

i. La mesure de la glycémie devrait être répétée si la première valeur obtenue est anormale ou si le patient a un diagnostic de diabète. L'hypoglycémie doit être corrigée immédiatement [niveau de preuve B] (AU, CSQCS, ESO).

ii. Les taux sensiblement élevés de glycémie devraient être traités à l'aide de médicaments réduisant l'hyperglycémie [niveau de preuve B] (AU, CSQCS, ESO).

\section{Recommandation 3.5 : Traitement thrombolytique aigu}

Il faut évaluer immédiatement tous les patients présentant un AVC ischémique aigu invalidant qui peuvent être traités dans les 4,5 heures suivant le déclenchement de leurs symptômes, afin de déterminer leur admissibilité à un traitement intraveineux par activateur du plasminogène tissulaire (tPA) (alteplase).

i. Les patients admissibles sont ceux qui peuvent recevoir de l'alteplase par voie intraveineuse dans les 4,5 heures qui suivent le début des symptômes d'AVC, conformément aux critères adaptés de l'étude portant sur le traitement de l'AVC par le tPA du National Institute of Neurological Disorders and Stroke (NINDS) et de la Third European Cooperative Acute Stroke Study (ECASS III) [niveau de preuve A] (Cochrane, ECASS III).

ii. Tous les patients admissibles doivent recevoir l'alteplase par voie i.v. dans l'heure qui suit leur arrivée à l'hôpital (délai entre l'arrivée et l'injection $<60$ minutes) [niveau de preuve C] (CSQCS, RCP).

iii. L'administration de l'alteplase se fait selon les lignes directrices de l'American Stroke Association : dose totale de $0,9 \mathrm{mg} / \mathrm{kg}$, dont $10 \%(0,09 \mathrm{mg} / \mathrm{kg})$ administrés en bolus intraveineux en 1 minute et les $90 \%$ restants $(0,81 \mathrm{mg} / \mathrm{kg})$ administrés sous forme de perfusion intraveineuse en 60 minutes [niveau de preuve A] (ASA, CSQCS, RCP).

iv. Les caractéristiques de la TDM cérébrale initiale qui modifieraient la réponse au traitement chez un patient victime d'AVC par ailleurs admissible au traitement par alteplase restent encore mal définies. Certains essais sur l'alteplase ont exclus les patients victimes d'un AVC hémisphérique grave si la TDM initiale montrait des signes précoces d'infarctus touchant plus du tiers du territoire irrigué par l'artère cérébrale moyenne (c.-à-d., un score inférieur à 5 à l'échelle ASPECTS [Alberta Stroke Program Early CT Score]). Dans la pratique clinique, la décision de traiter un tel patient par alteplase doit se fonder sur le jugement clinique du médecin traitant et sur les souhaits du patient et de sa famille, tant que l'on ne disposera pas d'autres données provenant d'études randomisées et contrôlées [niveau de preuve B] (Dzialowski et al. 2006) ${ }^{59}$.

v. Il reste des situations pour lesquelles les données tirées d'essais cliniques à l'appui du traitement thrombolytique sont rares, voire inexistantes : AVC chez l'enfant, AVC 
chez des patients de plus de 80 ans, adultes consultant au cours des quelques premières heures suivant le déclenchement d'un AVC ischémique aigu mais ne répondant pas aux critères actuels du traitement par alteplase intraveineux et par thrombolyse intra-artérielle. Dans la pratique clinique, la décision d'utiliser l'alteplase dans de telles circonstances doit se fonder sur le jugement clinique du médecin traitant et sur les souhaits du patient et de sa famille, tant que l'on ne disposera pas d'autres données provenant d'études randomisées et contrôlées [niveau de preuve A] (Cochrane, ECASS III, AHA-P).

Remarque : Au Canada, l'alteplase est actuellement approuvé par Santé Canada pour le traitement des adultes victimes d'un AVC ischémique aigu administré moins de trois heures après le début des symptômes d'AVC. Des exceptions s'appliquent, par exemple, une «lettre de non-objection» de Santé Canada est nécessaire pour les essais cliniques qui se penchent sur l'administration de l'alteplase par voie intraveineuse selon d'autres protocoles thérapeutiques.

\section{Recommandation 3.6 : Traitement actif par l'AAS}

Après que l'imagerie cérébrale a permis d'éliminer la possibilité d'une hémorragie intracrânienne, tous les patients victimes d'un AVC en phase aiguë devraient recevoir immédiatement une dose de charge unique d'au moins $160 \mathrm{mg}$ d'AAS [niveau de preuve A] (ESO, NZ, RCP, SIGN 13).

i. Chez les patients qui reçoivent un traitement par activateur recombinant du plasminogène, l'administration d'AAS devrait être reportée jusqu'à ce que la TDM effectuée 24 heures après la thrombolyse ait exclu l'hémorragie intracrânienne [niveau de preuve A] (NZ, $\mathrm{RCP})$.

ii. Le traitement par l'AAS (80 à $325 \mathrm{mg} /$ jour) devrait être poursuivi indéfiniment ou jusqu'à ce qu'un autre traitement antithrombotique soit amorcé [niveau de preuve A] (RCP). Consulter les recommandations 2.5 «Traitement antiplaquettaire» et 2.6 «Traitement antithrombotique pour la fibrillation auriculaire» pour plus de détails sur le traitement antiplaquettaire et l'anticoagulation.

iii. Chez les patients atteints de dysphagie, l'AAS peut être administré par voie intra-rectale ou par l'entremise d'un tube entérique [niveau de preuve A] (RCP).

iv. Chez les enfants, le traitement initial avec les héparines de bas poids moléculaire doit être envisagé et poursuivi jusqu'à ce que la dissection des artères vertébrales et le thrombus intracardiaque aient été exclus. En l'absence de ces deux cas, passer au traitement aigu par l'AAS à une dose de 3 à 5 mg/kg [niveau de preuve A] (AHA-P).

\section{Recommandation 3.7 : Prise en charge de l'hémor- ragie sous-arachnoïdienne et intracérébrale}

i. Il faudrait obtenir une consultation neurochirurgicale urgente pour établir le diagnostic et déterminer les soins de tous les patients chez qui une hémorragie sous-arachnoïdienne est soupçonnée [niveau de preuve B].

ii. Il faudrait obtenir une consultation neurochirurgicale urgente pour évaluer la nécessité d'une craniotomie pour l'évacuation de l'hémorragie de tous les patients d'hémorragie cérébelleuse [niveau de preuve C].

iii. Les patients avec hémorragie intracérébrale supratentorielle devraient être soignés dans une unité d'AVC [niveau de preuve B].

\section{4: Prise en charge de l'AVC en phase aiguë}

\section{Recommandation 4.1 : Soins en unité d'AVC}

Il faudrait soigner les patients hôpitalisés pour AVC aigu ou pour accident ischémique transitoire dans une unité interdisciplinaire d'AVC [niveau de preuve A] (CSQCS, ESO, SCORE, SIGN 64).

i. Une unité d'AVC est une unité spécialisée, géographiquement distincte dans un hôpital, et dédiée à la prise en charge des patients victimes d'AVC [niveau de preuve A] (AU, RCP).

ii. L'équipe interdisciplinaire de base devrait comprendre du personnel de niveaux appropriés de compétences dans les domaines suivants : médecine, soins infirmiers, ergothérapie, physiothérapie, orthophonie, travail social et nutrition clinique. Parmi les disciplines additionnelles, mentionnons la pharmacie, la (neuro)psychologie et la thérapie par les loisirs [niveau de preuve B] (AU, SCORE, SIGN 64).

iii. L'équipe interdisciplinaire devrait évaluer les patients dans les 48 heures qui suivent leur admission et préparer un plan de prise en charge [niveau de preuve C].

iv. Les cliniciens devraient utiliser des outils normalisés et valides pour mesurer les incapacités liées à l'AVC et l'état fonctionnel du patient [niveau de preuve B] (ASA, $\mathrm{RCP})$.

v. Tout enfant admis à l'hôpital en raison d'un AVC devrait être traité dans un centre compétent en soins de l'AVC chez l'enfant et pris en charge selon les protocoles normalisés relatifs à l'AVC chez l'enfant [niveau de preuve B] (ACCP, AHA-P, RCP-P).

\section{Recommandation 4.2 : Éléments des soins actifs chez le patient hospitalisé (nouvelle en 2008)}

Chez tous les patients hospitalisés pour AVC, il faut tenir compte des éléments suivants : risque de thromboembolie veineuse, température corporelle, mobilisation, continence, nutrition et soins de la bouche. Il faut appliquer les stratégies thérapeutiques appropriées aux problèmes détectés lors de l'examen de dépistage. La planification du congé fera partie de l'évaluation initiale et des soins continus chez les patients victimes d'un AVC aigu.

\section{2a Prophylaxie de la thromboembolie veineuse}

Chez tous les patients victimes d'AVC, il faut évaluer le risque de thromboembolie veineuse (y compris la thrombose veineuse profonde et l'embolie pulmonaire).

Les patients considérés à risque élevé sont ceux qui sont incapables de bouger l'un des deux membres inférieurs ou les deux et ceux qui sont incapables de se mouvoir seuls.

i. Il faut envisager le traitement prophylactique chez les patients jugés à risque élevé à l'égard de la thrombo- 
embolie veineuse, à moins de contre-indications [niveau de preuve $\mathrm{B}]$ (ESO).

ii. La mobilisation précoce et l'hydratation adéquate sont à encourager chez tous les patients victimes d'AVC aigu afin de contribuer à prévenir la thromboembolie veineuse [niveau de preuve C] (AU, ESO, SCORE).

iii. L'utilisation de mesures de prévention secondaire de l'AVC, comme le traitement antiplaquettaire, doit être optimisée chez tous les patients victimes d'AVC [niveau de preuve A] (ASA, AU, NZ, RCP, SIGN 13).

iv. On peut opter pour les interventions suivantes chez les patients victimes d'un AVC ischémique aigu exposés à un risque élevé de thromboembolie veineuse, en l'absence de contre-indications :

a. héparine de bas poids moléculaire (aux doses prophylactiques appropriées selon chaque agent) ou héparine aux doses prophylactiques (5 000 unités, deux fois par jour) [niveau de preuve A] (ASA, AU, ESO);

b. bas de compression [niveau de preuve B] (AU, ESO).

v. Pour les patients victimes d'un AVC hémorragique, des mesures prophylactiques non pharmacologiques (décrites plus haut) sont à envisager afin de réduire le risque de thromboembolie veineuse [niveau de preuve C].

\section{2b Température}

i. Lors des vérifications périodiques des signes vitaux, il faut mesurer la température corporelle (toutes les 4 heures pendant les 48 premières heures, puis selon le protocole de l'unité de soins ou le jugement clinique) [niveau de preuve C] (ESO).

ii. Si la température excède $37,5^{\circ} \mathrm{C}$, on augmentera la fréquence des vérifications et on amorcera la prise de mesures antipyrétiques (contre la fièvre) [niveau de preuve C] (ESO).

iii. Il faut traiter les causes de la fièvre et administrer des antipyrétiques afin de ramener la température à $<38^{\circ} \mathrm{C}$ chez les patients fébriles qui ont subi un AVC [niveau de preuve B] (ASA, CSQCS).

iv. En cas de fièvre, on recommande de rechercher une infection possible (foyer et cause) afin d'amorcer l'antibiothérapie qui s'impose [niveau de preuve C] (ESO).

\section{2c Mobilisation}

On définit la mobilisation comme la «capacité d'un patient de changer de position dans son lit, de s'asseoir, de se lever et possiblement de marcher» ${ }^{10}$.

i. Tous les patients hospitalisés pour un AVC aigu doivent être mobilisés aussi rapidement et aussi souvent que possible [niveau de preuve B] (AU) et préférablement au cours des 24 premières heures suivant le début de leurs symptômes d'AVC, à moins de contre-indication [niveau de preuve C] (CSQCS).

ii. Au cours des trois premiers jours suivant un AVC, il faut vérifier la tension artérielle, la saturation en oxygène et la fréquence cardiaque avant chaque mobilisation [niveau de preuve C] (AVERT).

iii. Tous les patients hospitalisés pour un AVC aigu doivent être évalués par des professionnels de la réadaptation le plus rapidement possible après leur admission [niveau de preuve $\mathrm{A}](\mathrm{RCP})$, préférablement au cours des 24 à 48 premières heures [niveau de preuve C] (NZ). Consulter la partie 5, «Réadaptation et réintégration en milieu communautaire», pour des recommandations connexes.

\section{2d Continence}

i. Il faut vérifier la présence d'incontinence ou de rétention urinaires (avec ou sans débordement) et d'incontinence fécale ou de constipation chez tous les patients victimes d'AVC [niveau de preuve C] (RNAO).

ii. Les patients victimes d'AVC qui souffrent d'incontinence urinaire doivent être évalués par un personnel dûment formé, à l'aide de critères d'évaluation fonctionnelle structurés [niveau de preuve B] (AU).

iii. L'utilisation de cathéters à demeure est à éviter. Le cas échéant, ces cathéters doivent être vérifiés quotidiennement et retirés le plus tôt possible [niveau de preuve C] (AU, CSQCS, RCP, VA/DoD).

iv. Il faut appliquer un programme d'entraînement vésical chez les patients qui souffrent d'incontinence urinaire [niveau de preuve C] (AU, VA/DoD).

v. L'utilisation d'un appareil à échographie portable est recommandée comme méthode indolore et non effractive à privilégier pour mesurer la quantité d'urine résiduelle après la miction et réduire ainsi le risque d'infection urinaire d'origine effractive ou de traumatisme urétral causé par le cathétérisme [niveau de preuve C] (CCF).

vi. Il faut appliquer un programme d'entraînement intestinal chez les patients victimes d'AVC qui souffrent de constipation opiniâtre ou d'incontinence fécale [niveau de preuve A] (VA/DoD).

\section{2e Nutrition}

i. Il faut vérifier l'équilibre nutritionnel et hydrique des patients victimes d'AVC au cours des 48 premières heures suivant leur admission, à l'aide d'un outil de vérification validé [niveau de preuve B] (AU, RPC, SIGN 78).

ii. Selon le cas, les résultats du processus de dépistage permettront d'orienter les patients vers un diététiste pour une évaluation plus approfondie et pour vérifier si une prise en charge prolongée de l'état nutritionnel et hydrique ne s'impose pas [niveau de preuve C] (NZ, SIGN 78).

iii. Les patients victimes d'un AVC chez qui on soupçonne des carences nutritionnelles ou hydriques, ou les deux, ce qui inclut la dysphagie, doivent être référés à un diététiste pour les actions suivantes :

a. des recommandations visant à combler les besoins nutritionnels et hydriques par voie orale, tout en encourageant des modifications à la texture des aliments ou à la consistance des liquides, selon l'évaluation par l'orthophoniste ou autre professionnel dûment formé [niveau de preuve C] (AU, SCORE);

b. un éventuel soutien nutritionnel par voie entérale (tube de gavage) dans les sept jours suivant l'admis- 
sion des patients qui sont incapables de s'alimenter et de boire suffisamment bien par la bouche. Cette décision doit être prise en collaboration avec l'équipe multidisciplinaire, les patients, leurs soignants et leur famille [niveau de preuve B] (AU, SIGN 78).

c. Consulter également la recommandation 6.1, «Évaluation de la dysphagie», pour la prise en charge de la dysphagie.

\section{2f Soins de la bouche}

i. Il faut soumettre tous les patients victimes d'AVC à un examen buccal et dentaire, ce qui inclut un dépistage des signes manifestes de maladie dentaire, l'évaluation de l'hygiène de la bouche et des appareils dentaires lors de l'admission ou peu après [niveau de preuve C] (Association dentaire canadienne).

ii. Pour les patients qui portent des prothèses dentaires complètes ou partielles, il faut déterminer si leurs habiletés motrices leur permettent de les porter en toute sécurité [niveau de preuve C].

iii. Un protocole de soins de la bouche approprié doit être utilisé chez chaque patient victime d'AVC, y compris chez ceux qui portent des prothèses [niveau de preuve C] (SIGN 78). Le protocole des soins de la bouche doit tenir compte de différents éléments, dont la fréquence des soins buccaux (deux fois par jour ou plus), les types de produits pour soins buccaux (pâte dentifrice, soie dentaire et rince-bouche), la prise en charge spécifique des patients atteints de dysphagie. Le protocole doit concorder avec les recommandations actuelles de l'Association dentaire canadienne [niveau de preuve B] (Association dentaire canadienne).

iv. Si l'application d'un protocole de soins de la bouche pose problème, il faut envisager une consultation en médecine dentaire, en ergothérapie, en orthophonie ou en hygiène dentaire [niveau de preuve $\mathrm{C}$ ].

v. Si l'on cerne des problèmes relatifs à la santé buccale ou aux prothèses dentaires, les patients doivent être référés à un dentiste pour consultation et prise en charge le plus rapidement possible [niveau de preuve $\mathrm{C}$ ].

\section{2g Planification du congé}

Il faut planifier le plus tôt possible le congé du patient après son admission à l'hôpital (service d'urgence ou unité de soins) [niveau de preuve B] (AU, RCP).

i. Il faut établir un processus pour assurer la participation des patients et des soignants à l'élaboration du plan de soins, à sa gestion et à la planification du congé [niveau de preuve $\mathrm{C}$ ].

ii. La communication entourant la planification du congé doit être maintenue tout au long de l'hospitalisation afin d'assurer une transition en douceur lorsque le patient quittera l'unité de soins aigus [niveau de preuve B] (AU, $\mathrm{RCP})$.

iii. Peu de temps après l'admission, il faut déjà fournir aux patients et aux soignants de l'information sur les enjeux entourant le congé et les besoins possibles des patients après leur départ de l'hôpital [niveau de preuve C].

\section{5 : Réadaptation et réintégration en milieu communautaire}

\section{Recommandation 5.1 : Évaluation initiale en vue de la réadaptation post-AVC}

Tous les patients victimes d'un AVC devraient être évalués afin de déterminer leurs besoins en matière de réadaptation.

i. Tous les patients victimes d'un AVC aigu qui sont hospitalisés devraient subir une évaluation initiale par des professionnels de la réadaptation aussitôt que possible après leur admission [niveau de preuve A] (RCP), de préférence dans les premières 24 à 48 heures. [niveau de preuve C] (NZ).

ii. Tous les patients victimes d'un AVC aigu qui ont des incapacités liées à l'AVC et qui ne sont pas admis à l'hôpital devraient subir une évaluation complète en clinique externe, pour dépister toute incapacité fonctionnelle. Cette évaluation comprend une évaluation cognitive, un dépistage de la dépression, une vérification de l'aptitude à conduire, de même que des évaluations fonctionnelles en vue d'un éventuel traitement de réadaptation [niveau de preuve $\mathrm{A}$ ] $(\mathrm{RCP})$, préférablement dans les deux semaines [niveau de preuve $\mathrm{C}$ ].

iii. Les cliniciens devraient utiliser des outils d'évaluation normalisés et valides pour évaluer les incapacités dues à l'AVC et l'état fonctionnel du patient [niveau de preuve C] (ASA, RCP-P). La version intégrale présente un tableau des outils recommandés.

iv. Il faudrait réévaluer les survivants d'un AVC grave ou modéré régulièrement pour déterminer leurs besoins en matière de réadaptation [niveau de preuve C] (HSFO).

Remarque : La réadaptation dite «externe» comprend les cliniques hospitalières de jour, les soins ambulatoires en clinique externe et la réadaptation à domicile.

\section{Recommandation 5.2 : Prestation de la réadaptation en milieu hospitalier du patient victime d'un AVC}

Tous les patients victimes d'un AVC qui sont hospitalisés et qui ont besoin de réadaptation devraient être soignés dans une unité d'AVC complète ou une unité de réadaptation postAVC par une équipe interdisciplinaire [niveau de preuve A] (AU-R).

i. Les soins de l'AVC après la phase aiguë devraient inclure des soins de réadaptation qui coordonnés et organisés [niveau de preuve A] (ASA).

ii. Tous les patients devraient être orientés vers une équipe spécialisée en réadaptation dans une unité géographiquement définie dès que possible après l'admission [niveau de preuve A] (RCP). Pour les enfants, les soins de l'AVC en phase aiguë et la réadaptation devraient être donnés dans une unité spécialisée en soins pour enfants [niveau de preuve B] (RCP-P).

iii. Les soins de l'AVC après la phase aiguë devraient être dispensés par des spécialistes de diverses disciplines expérimentés dans ce domaine de manière à assurer la cohérence et à réduire le risque de complications [niveau de preuve C] (RCP).

iv. L'équipe interdisciplinaire de réadaptation pourrait 
comprendre un médecin, une infirmière, un physiothérapeute, un ergothérapeute, un orthophoniste, un psychologue, un thérapeute en loisirs, le patient, la famille et les soignants [niveau de preuve A] (ASA). Pour les enfants, cela comprendrait aussi des éducateurs et des travailleurs de soutien pour enfants hospitalisés. Cette équipe interdisciplinaire de base devrait comprendre le niveau approprié de ces disciplines, tel que déterminé par la Stroke Unit Trialists' Collaboration [niveau de preuve B] (AHA-P, SIGN 64).

v. L'équipe interdisciplinaire de réadaptation devrait évaluer le patient en moins de 24 à 48 heures suivant l'admission et préparer un plan de réadaptation exhaustif tenant compte de la gravité de l'AVC et des besoins et objectifs du patient [niveau de preuve C] (HSFO, NZ).

vi. Il faudrait donner aux patients victimes d'un AVC modéré ou grave qui sont prêts pour la réadaptation et ont des objectifs de réadaptation l'occasion de participer à leur plan de réadaptation en milieu hospitalier [niveau de preuve A] (HSFO).

vii. L'équipe d'une unité d'AVC devrait tenir une réunion formelle interdisciplinaire au moins une fois par semaine pour évaluer les progrès et discuter des problèmes, des objectifs de la réadaptation et des arrangements en vue du congé des patients de l'unité [niveau de preuve B] (SIGN 64). Les plans de réadaptation individualisés devraient être mis à jour régulièrement en fonction des évaluations de l'état de santé des patients [niveau de preuve C].

viii. Les cliniciens devraient utiliser des outils d'évaluation valides normalisés pour déterminer les incapacités liées à l'AVC et l'état fonctionnel des patients [niveau de preuve B] (ASA, RCP).

ix. Lorsqu'il n'est pas possible d'admettre le patient dans une unité de réadaptation dédiée à l'AVC, une deuxième option moins favorable est la réadaptation de longue durée en établissement dans une unité de réadaptation mixte (c'est-à-dire une unité dans laquelle des soins interdisciplinaires sont fournis à des patients handicapés par divers troubles dont l'AVC) [niveau de preuve B] (SIGN 64).

\section{Recommandation 5.3 : Éléments de réadaptation en milieu hospitalier du patient victime d'un AVC}

Tous les patients ayant eu un AVC devraient commencer une réadaptation le plus tôt possible une fois leur état médical stabilisé [niveau de preuve A] (ASA).

i. Les patients devraient recevoir un traitement pertinent sur le plan clinique dont l'intensité et la durée nécessaires sont définies dans leur plan de réadaptation personnalisé et appropriées à leurs besoins et à leur niveau de tolérance [niveau de preuve A] (HSFO, RCP).

ii. Dans le contexte d'un plan de traitement personnalisé, les patients victimes d'un AVC devraient recevoir, au moins une heure de traitement direct administré par une équipe interprofessionnelle de traitement de l'AVC dans le cas de chaque traitement de base pertinent et ce, au moins cinq jours par semaine, selon leurs besoins et leur tolérance individuels [niveau de preuve A] (EBRSR). La durée du traitement doit dépendre de la gravité de l'AVC [niveau de preuve C] (EBRSR).

iii. L'équipe devrait promouvoir la pratique des habiletés acquises dans les activités courantes du patient de façon cohérente [niveau de preuve A] (RCP).

iv. Les traitements devraient comprendre l'utilisation répétitive et intense de nouvelles tâches qui incitent le patient à acquérir les habiletés motrices nécessaires pour se servir du membre affecté dans les tâches fonctionnelles et les activités [niveau de preuve A] (SCORE).

v. L'équipe d'une unité d'AVC devrait tenir une réunion formelle interdisciplinaire au moins une fois par semaine pour évaluer les progrès et discuter des problèmes, fixer les objectifs de la réadaptation et planifier le support aux patients après leur congé de l'unité [niveau de preuve B] (SIGN 64).

vi. Le plan de prise en charge devrait comporter une évaluation des besoins avant le congé afin d'assurer une transition sans heurt de la réadaptation à la communauté. Les éléments de planification du congé devraient inclure une visite à domicile par un professionnel de la santé, idéalement avant le congé, afin d'évaluer l'environnement familial, de déterminer si le patient peut réintégrer son foyer en toute sécurité, d'évaluer les besoins d'équipement et les modifications nécessaires et de commencer la formation des soignants quant aux activités instrumentales et autres activités de la vie quotidienne du patient dans son environnement [niveau de preuve $\mathrm{C}$ ].

\section{Recommandation 5.4 : Réadaptation ambulatoire et en milieu communautaire}

Après avoir obtenu leur congé de l'hôpital, les survivants d'AVC devraient continuer d'avoir accès à des soins spécialisés et à des services de réadaptation appropriés à leurs besoins (hôpital de soins actifs et(ou) établissement de réadaptation pour patients hospitalisés) [niveau de preuve A] (RCP).

i. Un congé précoce accompagné d'un suivi et une planification de la transition par une équipe interdisciplinaire de spécialistes, bien équipée et coordonnée est une solution de rechange acceptable à un plus long séjour dans une unité de soins de l'AVC. Il permet de réduire la durée du séjour en milieu hospitalier de patients sélectionnés [niveau de preuve A] (SIGN 64). Par contre, après un congé précoce, il ne faudrait pas que le suivi soit assumé par des services communautaires généraux (non spécifiques) [niveau de preuve A] (RCP).

ii. Les personnes qui éprouvent des difficultés à accomplir les activités de la vie quotidienne, y compris l'auto-administration de soins, la productivité et les loisirs, devraient recevoir des soins d'ergothérapie ou des soins multidisciplinaires ciblant les activités de la vie quotidienne [niveau de preuve A] (AU) [niveau de preuve $\mathrm{C}$ pour les enfants].

iii. Les interventions multifactorielles dans la communauté, y compris un programme d'exercice personnalisé, peuvent contribuer à prévenir les chutes et à atténuer leur gravité chez les personnes à risque de tomber [niveau de preuve A] (AU). 
iv. Il faudrait offrir aux personnes qui ont des problèmes de mobilité un programme d'exercice et les surveiller durant tout le programme [niveau de preuve B] (MacKayLyons et Howlett ${ }^{60}$, Pang et al. ${ }^{61}$ ).

v. Il faudrait enseigner aux patients aphasiques des techniques d'aide à la conversation [niveau de preuve A] (EBRSR).

vi. Il faudrait offrir aux patients atteints de dysphagie une thérapie portant sur la déglutition et une possibilité de réévaluation au besoin [niveau de preuve A] (Singh et Hamdy $^{62}$.

vii. Il faudrait offrir aux enfants victimes d'un AVC des conseils et des traitements qui visent à leur donner des habiletés reliées au jeu, à l'autogestion de la santé, aux loisirs et à l'école qui sont pertinentes sur le plan du développement et appropriées à leur environnements familial, communautaire et scolaire [niveau de preuve B] (Kirton et al..$^{63}$, RCP-P).

\section{Recommandation 5.5 : Suivi et réintégration dans la communauté}

Les personnes qui ont subi un AVC et vivent dans la communauté devraient faire l'objet d'une évaluation de suivi périodique et continue qui vise à déterminer leur rétablissement, à éviter que leur état se détériore et à maximiser le résultat fonctionnel.

i. Un fournisseur de soins primaires devrait suivre les patients qui ont été victimes d'un AVC aigu afin de s'attaquer aux facteurs de risque d'AVC, de répondre aux besoins de réadaptation continue et de poursuivre le traitement de comorbidités et d'autres séquelles de l'AVC [niveau de preuve C] (ASA).

ii. Les besoins individuels psychosociaux et de soutien des survivants de l'AVC et de leurs soignants devraient être réévalués périodiquement [niveau de preuve $\mathrm{A}$ ] (RCP).

iii. Les personnes vivant dans la communauté qui éprouvent des difficultés à accomplir les activités de la vie quotidienne devraient avoir accès à des services visant à améliorer les activités de la vie quotidienne ou à prévenir la détérioration de leurs capacités, selon le cas [niveau de preuve A] (AU).

iv. La recommandation 6.2, «Dépistage et prise en charge de la dépression post-AVC», devrait s'appliquer aussi dans le cadre du suivi et de l'évaluation des survivants de l'AVC dans la communauté [niveau de preuve C].

v. Les patients dont les activités continuent à être réduites six mois ou davantage après l'AVC devraient être évalués en vue de déterminer la réadaptation ciblée dont ils ont besoin [niveau de preuve A] (RCP)

vi. Les nourrissons et les enfants chez lesquels une nouvelle déficience motrice, linguistique ou cognitive fait son apparition avec le temps ont besoin d'un suivi d'évaluation continu tout au long de leur développement [niveau de preuve C] (AHA-P)

vii. Il faudrait évaluer continuellement, durant tout leur développement, les besoins sur le plan éducationnel et professionnel des enfants qui ont survécu à un AVC dans la communauté [niveau de preuve C] (AHA-P). viii. Il faudrait fournir aux survivants d'un $\mathrm{AVC}$ et à leur famille de l'information à jour et opportune conjuguée à des possibilités d'apprendre des membres de l'équipe interdisciplinaire et d'autres fournisseurs appropriés de services communautaires. La fourniture d'information n'est pas efficace à elle seule [niveau de preuve A] (AU)

ix. Il faudrait offrir aux patients et à leurs soignants des programmes d'éducation pour les aider à s'adapter à leur nouveau rôle [niveau de preuve B] (RCP).

\section{6 : Sujets choisis sur la prise en charge de I'AVC}

Cette partie est nouvelle pour la mise à jour de 2008. Elle comprend trois des recommandations originales de la version 2006 (évaluation de la dysphagie, dépression post-AVC et douleur à l'épaule) et une nouvelle recommandation sur l'atteinte cognitive d'origine vasculaire. Ces recommandations s'appliquent tout au long du continuum des soins de l'AVC, à partir du début des symptômes de l'AVC ou de l'accident ischémique transitoire, et elles doivent être prises en compte pendant le rétablissement à court terme. De plus, il faut veiller au dépistage et à la prise en charge de l'atteinte cognitive d'origine vasculaire et de la dépression post-AVC après la récupération qui suit la phase aiguë d'un AVC et le retour dans la communauté.

\section{Recommandation 6.1 : Évaluation de la dysphagie}

Chez les patients victimes d'un AVC, il faut vérifier la capacité de déglutir en utilisant un protocole simple, valide et fiable, applicable à leur chevet, dans le cadre de leur évaluation initiale et avant de commencer à leur administrer des médicaments, des liquides ou des aliments par la bouche [niveau de preuve B] (CSQCS, NZ, SCORE, SIGN 78).

i. Il faut surveiller étroitement les patients qui ne sont pas alertes dans les 24 premières heures suivant l'AVC et vérifier la présence de dysphagie, selon le tableau clinique [niveau de preuve $\mathrm{C}$ ].

ii. Les patients victimes d'AVC qui présentent des caractéristiques de dysphagie ou d'aspiration pulmonaire doivent subir un examen clinique complet de leur capacité de déglutition par un orthophoniste ou un spécialiste dûment formé qui pourrait donner son opinion, à savoir si le patient est capable d'avaler sans danger et s'il faut modifier la consistance des aliments et des liquides qu'il prend.[niveau de preuve A] (CSQCS, NZ, RCP, SCORE).

iii. On référera les patients exposés à un risque de malnutrition, y compris ceux qui souffrent de dysphagie, à un diététiste pour évaluation et traitement continu. L'évaluation du statut nutritionnel doit se faire à l'aide d'outils ou d'échelles de mesure validés appliqués à la nutrition [niveau de preuve C] (AU). Pour plus de détails, consulter la recommandation 4.2e, «Éléments des soins actifs chez le patient hospitalisé — Nutrition». 


\section{Recommandation 6.2 : Dépistage et prise en charge de la dépression post-AVC}

Il faudrait considérer que tous les patients victimes d'un AVC sont à risque élevé de dépression. Au moment de la première évaluation, l'équipe clinique doit déterminer si le patient a des antécédents de dépression ou s'il présente des facteurs de risque à cet égard [niveau de preuve B] (SCORE).

i. Il faut chercher à repérer chez tous les patients victimes d'un AVC des signes de dépression au moyen d'un outil validé [niveau de preuve A] (SCORE) (pour les outils recommandés, consulter la version intégrale). Le dépistage doit se faire à tous les points de transition et chaque fois qu'une manifestation clinique l'indique. Les points de transition peuvent inclure les suivants :

a. au moment de l'admission en soins actifs, particulièrement si l'on note des signes de dépression ou de changement d'humeur;

b. avant le congé du service de soins actifs ou pendant le début de la réadaptation si le patient est transféré dans un cadre de réadaptation en milieu hospitalier;

c. périodiquement au cours de la réadaptation en milieu hospitalier;

d. périodiquement à la suite du congé avec retour dans la communauté.

ii. Les patients jugés à risque de dépression à partir d'un outil de dépistage doivent être référés à un psychiatre ou à un psychologue qui poussera plus loin l'évaluation et le diagnostic [niveau de preuve B] (RCP, RCP-P).

iii. Il faut prendre en charge les patients qui présentent des symptômes dépressifs légers en assurant un suivi attentif et en commençant le traitement seulement si la dépression persiste [niveau de preuve A] (RCP).

iv. Les patients chez qui un trouble dépressif est diagnostiqué devraient faire l'objet d'un essai d'antidépresseurs, sauf s'ils sont contre-indiqués. On ne recommande pas une catégorie d'antidépresseurs plutôt qu'une autre; toutefois, tenant compte des effets secondaires, les inhibiteurs du recaptage de la sérotonine (IRS) seraient préférables dans cette population de patients [niveau de preuve A] (ASA).

v. Aux patients chez qui la tendance à pleurer est grave, persistante ou problématique, les antidépresseurs recommandés sont les IRS [niveau de preuve A] (ASA).

vi. Il faut offrir un suivi tout au long du traitement, qui doit durer au moins six mois, si l'on obtient de bons résultats [niveau de preuve $\mathrm{A}](\mathrm{RCP})$.

vii. Il faut chercher à dépister la présence d'un délire hypoactif chez tous les patients qui présentent des symptômes dépressifs apparents [niveau de preuve C].

viii. L'utilisation systématique d'antidépresseurs prophylactiques n'est pas recommandée après un AVC [niveau de preuve A] (ASA, RCP).

ix. Les patients devraient être renseignés, recevoir des conseils et avoir l'occasion de s'exprimer sur les répercussions de l'AVC sur leur vie [niveau de preuve B] (RCP).

x. Aux patients manifestement anxieux, une psychothérapie devrait être offerte [niveau de preuve B] (RCP). xi. Les besoins psychosociaux et de soutien particuliers des patients et de leurs soignants devraient être régulièrement révalués dans le cadre du rétablissement et de la gestion à long terme de l'AVC [niveau de preuve A] (RCP).

\section{Recommandation 6.3 : Atteinte cognitive et démence d'origine vasculaire (nouvelle pour 2008)}

Il faut considérer que tous les patients présentant des facteurs de risque vasculaires et ceux qui ont été victimes d'un AVC ou d'un accident ischémique transitoire cliniquement manifeste sont exposés à un risque élevé d'atteinte cognitive d'origine vasculaire.

Les patients considérés à risque élevé d'atteinte cognitive et perceptuelle sont ceux qui présentent des facteurs de risque vasculaire, dont les suivants : hypertension, âge $>65$ ans, hyperlipidémie, diabète, AVC clinique, signes d'AVC silencieux ou de maladie de la substance blanche aux épreuves de neuro-imagerie, atteinte d'autres organes cibles et(ou) anomalies cognitives ou fonctionnelles cliniquement manifestes ou signalées lors de l'anamnèse.

\section{3a Évaluation}

i. Il faut soumettre tous les patients mentionnés plus haut à un dépistage de l'atteinte cognitive à l'aide d'un outil validé [niveau de preuve B] (AU, SCORE). La version intégrale présente un tableau des outils recommandés.

ii. Le dépistage de l'atteinte cognitive chez un patient doit porter sur les domaines suivants : niveau d'éveil, niveau de vigilance, niveau d'attention, orientation, mémoire, langage, agnosie, fonction visuospatiale et perceptuelle, praxie et fonctions exécutives, telles que l'introspection, le jugement, la cognition sociale, la résolution de problèmes, le raisonnement abstrait, l'esprit d'initiative, la planification et l'organisation [niveau de preuve $\mathrm{C}$ ].

iii. Le questionnaire MoCA (Montreal Cognitive Assessment) est jugé plus sensible pour l'atteinte cognitive que le mini-examen de l'état mental chez les patients souffrant d'une atteinte cognitive d'origine vasculaire. Son utilisation est recommandée lorsqu'on soupçonne une atteinte cognitive d'origine vasculaire [niveau de preuve B] (CCCDTD). Une validation additionnelle est requise pour le test MoCA et il faut d'autres instruments de dépistage, comme le protocole de 5 minutes qui figure dans les normes d'harmonisation pour l'évaluation de l'atteinte cognitive d'origine vasculaire.

iv. Il faut également effectuer un dépistage de la dépression chez les patients, puisque la dépression s'est révélée susceptible de contribuer à l'atteinte cognitive chez les patients victimes d'AVC. Il faut utiliser un outil de dépistage validé pour la dépression [niveau de preuve $\mathrm{B}$ ] (CCCDTD). Consulter la recommandation 6.2, «Dépistage et prise en charge de la dépression postAVC».

v. On fera subir des évaluations cognitives ou neuropsychologiques plus approfondies, selon le cas, chez les personnes qui présentent une atteinte cognitive lors d'un test de dépistage, pour mieux orienter la prise en charge [niveau de preuve B] (CCCDTD). 


\section{3b Synchronisation}

i. Selon la gravité du tableau clinique, il faudra évaluer périodiquement l'historique et(ou) les anomalies à l'imagerie, chez tous les patients jugés à risque élevé à l'égard d'une atteinte cognitive pour déceler les déficits perceptuels et cognitifs, la dépression, le délire ou les anomalies fonctionnelles [niveau de preuve C].

ii. Les sujets qui ont subi un accident ischémique transitoire ou un AVC doivent subir une évaluation de dépistage et, selon le cas, une évaluation plus approfondie de leur état cognitif et perceptuel aux différentes étapes de transition tout au long du continuum des soins de l'AVC [niveau de preuve C]. Les étapes de transition peuvent inclure :

a. le moment de la consultation à l'urgence, lorsque des problèmes cognitifs, perceptuels ou fonctionnels sont notés;

b. le moment de l'admission à l'unité de soins actifs, particulièrement en présence de signes de délire;

c. le moment du congé vers le domicile au départ de l'unité des soins actifs ou durant la période initiale de réadaptation, si le patient est transféré dans un centre de réadaptation à titre de patient hospitalisé;

d. périodiquement durant la phase de réadaptation en cours d'hospitalisation, selon les progrès du patient, et pour aider à la planification du congé;

e. périodiquement après le congé pour retourner dans la communauté; les évaluations seront alors faites par le professionnel de la santé dans la communauté, selon les besoins, les progrès et les objectifs courants du patient.

\section{3c Prise en charge}

i. Il faut gérer énergiquement tous les facteurs de risque vasculaire pour atteindre une maîtrise optimale [niveau de preuve A] (CCCDTD). Consulter également la partie 2 «Prévention de l'AVC».

ii. Les patients qui manifestent des atteintes cognitives lors du processus de dépistage doivent être référés à un professionnel de la santé détenant des connaissances précises dans ce domaine pour une évaluation cognitive, perceptuelle ou fonctionnelle plus approfondie afin de déterminer la gravité de l'atteinte et l'impact des déficiences sur le fonctionnement et la sécurité du patient lors des activités, instrumentales et autres, de la vie quotidienne et pour appliquer les mesures correctives et les stratégies d'intervention compensatoires ou adaptatives qui s'imposent [niveau de preuve B] (CCCDTD). On recommande une approche multidisciplinaire regroupant, entre autres professionnels de la santé, un ergothérapeute, un neuropsychologue, un psychiatre, un psychologue, un gériatre, un orthophoniste ou un travailleur social.

iii. Une approche individualisée, axée sur le patient est à envisager pour faciliter la reprise des activités souhaitées, comme le retour au travail, les activités de loisirs, le bénévolat, la gestion des finances personnelles, les tâches domestiques et autres activités instru- mentales de la vie quotidienne [niveau de preuve C] (CCCDTD).

iv. Les stratégies d'intervention incluant la réadaptation doivent être adaptées selon les atteintes cognitives et les restrictions fonctionnelles et selon les capacités cognitives restantes repérées dans le cadre d'une évaluation en profondeur et conçues en fonction des besoins et des objectifs des patients et des soignants [niveau de preuve B] (SCORE)

v. Un entraînement stratégique ou compensatoire semble être efficace pour le traitement de l'apraxie post-AVC. C'est pourquoi il faut l'envisager [niveau de preuve A] (EBRSR). Les preuves de l'efficacité des interventions précises axées sur l'atteinte cognitive résultant d'un AVC sont limitées et devraient faire l'objet de recherches plus approfondies. Un entrainement axé sur l'attention pourrait avoir un effet positif sur certains paramètres spécifiques et ciblés et doit être appliqué chez les patients appropriés [niveau de preuve C] (EBRSR). Les stratégies compensatoires peuvent être utilisées pour améliorer les paramètres liés à la mémoire [niveau de preuve C] (EBRSR).

vi. Les patients qui présentent des signes de dépression ou d'anxiété lors du dépistage doivent être vus et pris en charge par un professionnel de la santé mentale dûment formé [niveau de preuve $\mathrm{C}$ ].

vii. Pharmacothérapie :

a. On doit adresser les patients qui présentent des signes d'atteinte cognitive d'origine vasculaire à un médecin expert de ces atteintes pour une évaluation plus approfondie et des recommandations quant au traitement pharmacologique [niveau de preuve $\mathrm{C}$ ].

b. On peut envisager les inhibiteurs de la cholinestérase pour la prise en charge de l'atteinte cognitive d'origine vasculaire diagnostiquée à l'aide des critères diagnostiques du National Institute of Neurological Disorders and Stroke (NINDS) et de l'Association internationale pour la recherche et l'enseignement en neurosciences (AIREN) [niveau de preuve B] (CCCDTD).

c. On dispose de preuves acceptables selon lesquelles la galantamine entraîne des résultats légèrement positifs sur la fonction cognitive et le comportement dans la maladie d'Alzheimer et la maladie vasculaire cérébrale mixte. La galantamine peut aussi être envisagée comme option thérapeutique pour la maladie d'Alzheimer et la maladie vasculaire cérébrale mixte [niveau de preuve B] (CCCDTD).

d. On dispose de preuves acceptables selon lesquelles le donépézil entraîne des résultats légèrement positifs sur les paramètres cognitifs et globaux avec des avantages moins marqués sur les paramètres fonctionnels. Le donépézil peut être envisagé comme option thérapeutique pour la démence vasculaire [niveau de preuve B] (CCCDTD).

Remarque : On peut également consulter la recommandation 6.2 «Dépistage et prise en charge de la dépression postAVC». 


\section{Recommandation 6.4 : Évaluation et soins de la douleur à l'épaule}

Il faudrait évaluer tous les patients victimes d'AVC pour la douleur à l'épaule et, en présence de symptômes, mettre en place des stratégies pour réduire au minimum la douleur à l'épaule et les traumatismes articulaires [niveau de preuve A] (Groupe d'Ottawa, RCP, SCORE).

i. Les facteurs qui contribuent à la douleur à l'épaule ou l'exacerbent doivent être déterminés et gérés de manière appropriée.

a. Le personnel et les soignants doivent recevoir une éducation sur la manière appropriée de manipuler un bras hémiplégique [niveau de preuve B] (RCP, SCORE).

b. Il faudrait envisager l'utilisation d'une écharpe pour le bras [niveau de preuve A] (RCP).

ii. Des stratégies de protection des articulations devraient être adoptées pour réduire au minimum les traumatismes articulaires.

a. L'épaule ne devrait pas être passivement manipulée au-delà de 90 degrés de flexion et d'abduction à moins que l'omoplate ne soit tournée vers le haut et l'humérus pivoté latéralement [niveau de preuve A] (SCORE).

b. Les poulies verticales ne devraient pas être utilisées [niveau de preuve A] (Groupe d'Ottawa).

c. Le membre supérieur doit être manipulé avec soin lors des activités fonctionnelles [niveau de preuve B] (SCORE).

d. Le personnel devrait positionner les patients, qu'ils soient couchés ou assis, de manière à réduire au minimum le risque de complications telles que la douleur à l'épaule [niveau de preuve $\mathrm{B}$ ] (RCP).

iii. La douleur à l'épaule et les limitations dans l'amplitude des mouvements devraient être soignées par des techniques douces d'étirement et de mobilisation axées en particulier sur la rotation et l'abduction externe [niveau de preuve B] (SCORE).

Ce document a fait l'objet d'un examen critique par des pairs.

Intérêts concurrents : Seize participants à l'exercice de production et de critique des recommandations ont reçu des subventions de recherche ou des honoraires liés à l'accident vasculaire cérébral. On a jugé qu'aucun des intérêts déclarés n'empêchait les intéressés de participer au processus en toute impartialité. Voir les Annexes 1 et 2 de cette version abrégée ainsi que les annexes supplémentaire de la version intégrale concernant les déclarations complètes d'intérêts concurrents. Le $J A M C$ a été rémunéré par le Réseau canadien contre les accidents cérébrovasculaires pour ce supplément et il l'a soumis à un examen critique par des pairs.

Collaborateurs : Les Recommandations canadiennes pour les pratiques optimales de soins de l'AVC (mise à jour de novembre 2008) ont été créées grâce à la collaboration de plus de 150 spécialistes et intervenants en AVC. La préparation de la version définitive du document incombait à un groupe de rédaction.

$\mathrm{M}^{\text {me }}$ Patrice Lindsay, $\mathrm{PhD}$, a agi à titre de chef de projet et s'est chargée du processus global d'élaboration des recommandations et des documents d'appui, y compris la création et la révision des éléments de chaque modèle de recommandation ainsi que de la collecte et de la révision des travaux de chaque groupe de travail pour chacune des recommandations. La publication de la version intégrale et de la version abrégée imprimée a aussi été placée sous sa responsabilité. Le $\mathrm{D}^{\mathrm{r}}$ Mark Bayley a apporté une contribution signi- ficative à l'ensemble du processus de formulation des recommandations. Il a participé à la création et à la rédaction des éléments sur la réadaptation et sur les sujets choisis, ainsi qu'à la révision des premières ébauches de la version intégrale du document. $\mathrm{M}^{\mathrm{me}}$ Chelsea Hellings a coordonné le projet. Elle a élaboré et préparé tous les résumés des preuves et a contribué à la préparation d'autres composantes des modèles de recommandation et des annexes. Le $\mathrm{D}^{r}$ Michael Hill était chargé de l'élaboration et de la révision des indicateurs de rendement et des notes relatives à la mesure des indicateurs. Il a aussi apporté une contribution importante aux éléments sur les soins actifs et la prévention. $\mathrm{M}^{\mathrm{me}}$ Elizabeth Woodbury a contribué à la création et à la rédaction de l'aperçu et des annexes, ainsi qu'à la révision des premières ébauches de la version intégrale. Le $\mathrm{D}^{\mathrm{r}}$ Stephen Phillips était l'auteur principal de ces lignes directrices. Il a contribué de manière significative à la création et à la rédaction des éléments sur les soins actifs et sur la prévention, ainsi qu'à la révision des multiples ébauches de la version intégrale. Tous les membres du groupe de rédaction ont approuvé la version définitive du document pour publication.

Remerciements : Nous remercions sincèrement les chefs et les membres des groupes de travail, le comité de consensus et les examinateurs externes, qui ont tous contribué bénévolement leur temps et leurs connaissances tout au long de l'exercice rigoureux de production des recommandations. Nous remercions le Groupe de travail sur l'information et l'évaluation de la Stratégie canadienne de l'AVC d'avoir participé à l'exercice pour actualiser et confirmer les indicateurs de rendement joints à chaque recommandation. Nous remercions $\mathrm{M}^{\mathrm{me}}$ Gail Williams qui a mis à profit ses compétences professionnelles pour la révision du document avant sa présentation pour publication. Nous tenons également à exprimer notre reconnaissance au $\mathrm{D}^{\mathrm{r}}$ Sylvain Lanthier, de Montréal, et à $\mathrm{M}^{\mathrm{me}}$ Nicole Pageau, de Mississauga, qui ont généreusement consacré temps, attention et effort à la vérification de la traduction française de ces recommandations sur l'AVC.

Financement : La Stratégie canadienne de l'AVC est une initiative conjointe du Réseau canadien contre les accidents cérébrovasculaires et de la Fondation des maladies du cœur du Canada. La Stratégie canadienne de l'AVC a financé intégralement la production du présent document. Les fonds proviennent principalement du Réseau canadien contre les accidents cérébrovasculaires, qui est financé par le programme des Réseaux de centres d'excellence. Aucun fonds pour le présent document ne provient d'intérêts commerciaux, y compris des sociétés pharmaceutiques.

\section{RÉFÉRENCES}

1. Réseau canadien contre les accidents cérébrovasculaires et Fondation des maladies du cœur du Canada. Recommandations canadiennes pour les pratiques optimales de soins de l'AVC : 2006. Ottawa (Ont.) : La Stratégie canadienne de l'AVC; 2006. Disponible : www.canadianstrokestrategy.ca/fr/outilsressources/documents/StrokeStrategyManual_fr.pdf (consulté le 20 octobre 2008).

2. Graham ID, Harrison MB, Lorimer K, et al. Adapting national and international leg ulcer practice guidelines for local use: the Ontario Leg Ulcer Community Care Protocol. Adv Skin Wound Care 2005;18:307-18.

3. Adams HP Jr, del Zoppo G, Alberts MJ, et al. Guidelines for the early management of adults with ischemic stroke. A guideline from the American Heart Association/American Stroke Association Stroke Council, Clinical Cardiology Council, Cardiovascular Radiology and Intervention Council, and the Atherosclerotic Peripheral Vascular Disease and Quality of Care Outcomes in Research Interdisciplinary Working Group. Stroke 2007;38:1655-711.

4. Sacco RL, Adams R, Albers G, et al. Guidelines for prevention of stroke in patients with ischemic stroke or transient ischemic attack: a statement for healthcare professionals from the American Heart Association/American Stroke Association Council on Stroke. Stroke 2006;37:577-617.

5. Goldstein LB, Adams R, Alberts MJ, et al. Primary prevention of ischemic stroke. A guideline from the American Heart Association/American Stroke Association Stroke Council. Stroke 2006;37:1583-633.

6. Broderick J, Connolly S, Feldmann E, et al. Guidelines for the management of spontaneous intracerebral hemorrhage in adults: 2007 update: a guideline from the American Heart Association/American Stroke Association Stroke Council, High Blood Pressure Research Council, and the Quality of Care and Outcomes in Research Interdisciplinary Working Group. Stroke 2007;38:2001-23.

7. Duncan PW, Zorowitz R, Bates B, et al. Management of adult stroke rehabilitation care: a clinical practice guideline. Stroke 2005;36:e100-43.

8. Roach ES, Golomb MR, Adams R, et al. Management of stroke in infants and children: a scientific statement from a special writing group of the American Heart Association Stroke Council and the Council on Cardiovascular Disease in the Young. Stroke 2008;39:2644-91.

9. Hirsh J, Guyatt G, Albers GW, et al. Executive summary: American College of Chest Physicians evidence-based clinical practice guidelines (8th edition). Chest 2008;133(Suppl):71S-109S

10. Veterans Affairs/Department of Defense, Office of Quality Performance. VA/DoD 
clinical practice guideline for management of stroke rehabilitation. Washington (DC) : US Department of Veterans Affairs; 2003. Disponible : www.oqp.med.va gov/cpg/STR/str_cpg/frameset.htm (consulté le 23 octobre 2008).

11. National Stroke Foundation. Clinical guidelines for acute stroke management Melbourne (AU) : The Foundation; 2007. Disponible : www.strokefoundation .com.au/acute-clinical-guidelines-for-Acute-stroke-management (consulté le 20 octobre 2008).

12. National Stroke Foundation. Clinical guidelines for stroke rehabilitation and recovery. Melbourne (AU) : The Foundation; 2005. Disponible : www.strokefoundation.com.au/post-acute-health-professional (consulté le 20 octobre 2008).

13. Chertkow H. Introduction: the Third Canadian Consensus Conference of the Diagnosis and Treatment of Dementia 2006. Alzheimers Dement 2007;3:262-5.

14. Canadian Diabetes Association Clinical Practice Guidelines Expert Committee. Canadian Diabetes Association 2008 clinical practice guidelines for the prevention and management of diabetes in Canada. Can J Diabetes 2008;32(Suppl 1):S1-201.

15. McPherson R, Frohlich J, Fodor G, et al. Guidelines for the diagnosis and treat ment of dyslipidemia and prevention of cardiovascular disease: recommendations for the diagnosis and treatment of dyslipidemia and prevention of cardiovascular disease. Can J Cardiol 2006;22:913-27.

16. Khan NA, Hemmelgarn B, Herman RJ, et al.; Canadian Hypertension Education Program. The 2008 Canadian Hypertension Education Program recommendations for the management of hypertension: part 2 - therapy. Can J Cardiol 2008;24:465-75.

17. Lindsay P, Kapral M, Holloway R, et al. Canadian Stroke Quality of Care Study: identification of performance indicators for acute stroke care. CMAJ 2005;172(3) : Online-1-8. Available : www.cmaj.ca/cgi/content/full/172/3/363/DC1 (accessed 2008 Nov 3).

18. Lindsay P, Jaigobin C, Silver FL, et al. Canadian Stroke Quality of Care Study: quality indicators and literature review for telestroke. Toronto (Ont) : Réseau canadien contre les accidents cérébrovasculaires, ministère de la Santé et des Soins de longue durée de l'Ontario et Institut de recherche en services de santé (Ontario); 2005 .

19. Lindsay P, Gladstone DJ, Silver FL, et al. Canadian Stroke Quality of Care Study: Quality indicators and literature review for stroke secondary prevention. Toronto (Ont) : Réseau canadien contre les accidents cérébrovasculaires, ministère de la Santé et des Soins de longue durée de l'Ontario et Institut de recherche en services de santé (Ontario); 2005.

20. Bayley M, Lindsay P, Teasell R, et al. Canadian Stroke Quality of Care Study: Quality indicators for stroke rehabilitation (in collaboration with SCORE). Toronto (Ont) : Réseau canadien contre les accidents cérébrovasculaires, ministère de la Santé et des Soins de longue durée de l'Ontario et Institut de recherche en services de santé (Ontario); 2006.

21. Fondation des maladies du cœur de l'Ontario. Best Practice guidelines for stroke care. Toronto (Ont.) : La Fondation; 2003. Disponible : http://profed.heartandstroke.ca/Page.asp?PageID=397\&CategoryID=77 (consulté le 28 octobre 2008).

22. Association des infirmières et infirmiers autorisés de l'Ontario. Nursing best practice guidelines. Stroke assessment across the continuum of care. Toronto (Ont.) L'Association; 2005. Disponible : www.rnao.org/Storage/12/652_BPG_Stroke _Assessment.pdf (consulté le 20 octobre 2008)

23. Ottawa Panel, Khadilkar A, Phillips K, et al. Ottawa Panel evidence-based clinical practice guidelines for post-stroke rehabilitation. Top Stroke Rehabil 2006;13:1-269.

24. Teasell R, Foley N, Salter K, et al. EBRSR: evidence-based review of stroke rehabilitation. $10^{\mathrm{e}}$ édition. London (Ont.) : EBRSR; 2007. Disponible : www.EBRSR com (consulté le 20 octobre 2008).

25. European Stroke Organization (ESO) Executive Committee, ESO Writing Committee. Guidelines for the management of ischaemic stroke and transient ischaemic attack 2008. Cerebrovasc Dis 2008:25:457-507.

26. Stroke Foundation of New Zealand. Life after stroke: New Zealand guideline for management of stroke. Wellington (NZ) : The Foundation; 2003. Disponible www.nzgg.org.nz/guidelines/0037/ACF291F.pdf (consulté le 20 octobre 2008).

27. Scottish Intercollegiate Guidelines Network. Management of patients with stroke I Assessment, investigation, immediate management and secondary prevention (SIGN 13). Edinburgh (RU) : The Network; 1997. Disponible : www.sign.ac.uk /pdf/sign13.pdf (consulté le 20 octobre 2008).

28. Scottish Intercollegiate Guidelines Network. Management of patients with stroke II: Management of carotid stenosis and carotid endarterectomy (SIGN 14). Edinburgh (RU) : The Network; 1997. Disponible : www.sign.ac.uk/pdf/sign14.pdf (consulté le 20 octobre 2008)

29. Scottish Intercollegiate Guidelines Network. Management of patients with stroke III: Identification and management of dysphagia (SIGN 78). Edinburgh (RU) The Network; 2004. Disponible : www.sign.ac.uk/pdf/sign78.pdf (consulté le 20 octobre 2008).

30. Scottish Intercollegiate Guidelines Network. Management of patients with stroke $I V$ : Rehabilitation, prevention and management of complications and discharge planning (SIGN 64). Edinburgh (RU) : The Network; 2005. Disponible : www .sign.ac.uk/pdf/sign64.pdf (consulté le 20 octobre 2008)

31. National Collaborating Centre for Chronic Conditions. Stroke: diagnosis and initial management of acute stroke and transient ischaemic attack (TIA). London (RU) : National Institute for Health and Clinical Excellence; 2008. Disponible www.nice.org.uk/nicemedia/pdf/CG68NICEGuideline.pdf (consulté le 20 octobre 2008).

32. National Institute for Health and Clinical Excellence. Alteplase for the treatment of acute ischaemic stroke. London (RU) : The Institute; 2007. Disponible : www.nice.org.uk/nicemedia/pdf/TA122guidance.pdf (consulté le 20 octobre 2008).
33. National Collaborating Centre for Chronic Conditions. Atrial fibrillation: the management of atrial fibrillation. London (RU) : National Institute for Health and Clinical Excellence; 2006. Disponible : www.nice.org.uk/nicemedia/pdf /CG036niceguideline.pdf (consulté le 20 octobre 2008).

34. National Institute for Health and Clinical Excellence. Clopidogrel and modified-release dipyridamole in the prevention of occlusive vascular events. London (RU) The Institute; 2005. Disponible : www.nice.org.uk/nicemedia/pdf/TA090guidance .pdf (consulté le 20 octobre 2008).

35. Royal College of Physicians of London, Intercollegiate Stroke Working Party Clinical Effectiveness and Evaluation Unit. National clinical guidelines for stroke. 3rd ed. London (RU) : The College; 2008.

36. National Pre-Hospital Guidelines Group. The recognition and emergency management of suspected stroke and TIA guidelines supplement. London (RU) : Royal College of Physicians; 2006.

37. Paediatric Stroke Working Group. Stroke in childhood: clinical guidelines for diagnosis, management and rehabilitation. London (RU) : Royal College of Physicians; 2004. Disponible : www.rcplondon.ac.uk/pubs/books/childstroke/childstroke_guidelines.pdf (consulté le 20 octobre 2008).

38. SCORE. (Stroke Canada optimization of rehabilitation through evidence) evidence-based recommendations for the upper and lower extremities and risk assessment post-stroke 2007. Ottawa (Ont.) : Réseau canadien contre les accidents cérébrovasculaires; 2007. Disponible : www.canadianstrokenetwork.ca/eng/tools /downloads/SCORE_EBR_Aug2307.pdf (consulté le 28 octobre 2008).

39. Chaturvedi S, Bruno A, Feasby T. Carotid endarterectomy — an evidence-based review: report of the Therapeutics and Technology Assessment Subcommittee of the American Academy of Neurology. Neurology 2005;65:794-801.

40. Bernhardt J, Dewey H, Collier J, et al. A Very Early Rehabilitation Trial (AVERT). Int J Stroke 2006;1:169-71.

41. Bernhardt J, Dewey H, Thrift A, et al. A Very Early Rehabilitation Trial for stroke (AVERT): phase II safety and feasibility. Stroke 2008;39:390-6.

42. CAST (Chinese Acute Stroke Trial) Collaborative Group. CAST: randomised placebo-controlled trial of early aspirin use in 20,000 patients with acute ischaemic stroke. Lancet 1997;349:1641-9.

43. Fondation d'aide aux personnes incontinentes. Guide des pratiques cliniques chez les adultes. London (Ont.) : La Fondation; 2004. Disponible : www.canadiancontinence.ca/french/health-profs/clinicalpractice_fr.html (consulté le 20 octobre 2008).

44. Bhatt DL, Fox KA, Hacke W, et al. Clopidogrel and aspirin versus aspirin alone for the prevention of atherothrombotic events. N Engl J Med 2006;354:1706-17.

45. Wardlaw JM, Murray V, Sandercock PAG. Thrombolysis for acute ischaemic stroke: an update of the Cochrane thrombolysis meta-analysis [résumé]. Int J Stroke 2008:3(1 Suppl);50. Voir aussi le diaporama d'accompagnement, «Presentation by Johanna Wardlaw», Disponible : www.dcn.ed.ac.uk/ist3/ (consulté le 23 octobre 2008)

46. Hacke W, Kaste M, Bluhmki E, et al. ECASS Investigators. Thrombolysis with alteplase 3 to 4.5 hours after acute ischemic stroke. N Engl J Med 2008;359:1317-29.

47. Rothwell PM, Giles MF, Chandratheva A, et al. Effect of urgent treatment of transient ischaemic attack and minor stroke on early recurrent stroke (EXPRESS study): a prospective population-based sequential comparison. Lancet 2007; 370:1432-42.

48. Consensus Panel on the Stroke Rehabilitation System to the Ministry of Health and Long-Term Care. Consensus Panel on the Stroke Rehabilitation System: time is function. Toronto (Ont.) : Fondation des maladies du cœur de l'Ontario; 2007.

49. Diener HC, Bogousslavsky J, Brass LM, et al. Aspirin and clopidogrel compared with clopidogrel alone after recent ischaemic stroke or transient ischaemic attack in high-risk patients (MATCH): randomised, double-blind, placebo-controlled trial. Lancet 2004;364:331-7.

50. National Association of EMS Physicians. Role of EMS in the management of acute stroke: triage, treatment and stroke systems. Lenexa (KS) : The Association; 2007. Disponible : www.naemsp.org/position.html (consulté le 20 octobre 2008).

51. Association des Paramédics du Canada. National occupational competency profiles for paramedic practitioners. Kamloops (C.-B.) : L'Association; 2001. Disponible : www.paramedic.ca (consulté le 20 octobre 2008).

52. Lau DCW, Douketis JD, Morrison KM, et al. 2006 Canadian clinical practice guidelines on the management and prevention of obesity in adults and children. CMAJ 2007;176 (8 Suppl):Online-1-117. Available : www.cmaj.ca/cgi/data/176 /8/S1/DC1/1 (consulté le 3 november 2008).

53. PROGRESS Collaborative Group. Randomised trial of a perindopril-based bloodpressure-lowering regimen among 6,105 individuals with previous stroke or transient ischaemic attack. Lancet 2001;358:1033-41.

54. Guyatt GH, Cook DJ, Jaeschke R, et al. Grades of recommendation for antithrombotic agents: American College of Chest Physicians evidence-based clinical practice guidelines (8th edition) [errata publié dans Chest 2008;134:473]. Chest 2008; 133(Suppl):123S-31S.

55. Cameron JI, Gignac MA. "Timing it right": a conceptual framework for addressing the support needs of family caregivers to stroke survivors from the hospital to the home. Patient Educ Couns 2008;70:305-14.

56. Hare R, Rogers H, Lester H, et al. What do stroke patients and their carers want from community services? Fam Pract 2005;23:131-6.

57. Glossaire de la promotion de la santé. Genève : Organisation mondiale de la santé; 1998. Disponible : www.who.int/hpr/NPH/docs/ho_glossary_fr.pdf (consulté le 28 octobre 2008).

58. Stroke: prevention. Dans : Fondation des maladies du cœur du Canada [site web]. Toronto (Ont.) : La Fondation. Disponible : http://profed.heartandstroke.ca (choisir «Stroke», puis «Stroke care resources»).

59. Dzialowski I., Hill MD., Coutts SB., et al. Extent of early ischemic changes on 
computed tomography (CT) before thrombolysis: prognostic value of the Alberta Stroke Program Early CT Score in ECASS II. Stroke 2006;37:973-8.

60. MacKay-Lyons MJ, Howlett J. Exercise capacity and cardiovascular adaptations to aerobic training early after stroke. Top Stroke Rehabil 2005;12:31-44.

61. Pang MY, Eng JJ, Dawson AS, et al. The use of aerobic exercise training in improving aerobic capacity in individuals with stroke: a meta-analysis. Clin Rehabil 2006;20:97-111.

62. Singh S, Hamdy S. Dysphagia in stroke patients. Postgrad Med J 2006;82:383-91.

63. Kirton A, Chen R, Friefeld S, et al. Contralesional repetitive transcranial magnetic stimulation for chronic hemiparesis in subcortical paediatric stroke: a randomised trial. Lancet Neurol 2008;7:507-13.

Correspondance : $M^{m e} M$. Patrice Lindsay, PhD, Réseau canadien contre les accidents cérébrovasculaires, 600 prom. Peter Morand, bureau 301, Ottawa ON K1G $5 Z 3$ (téléc. : 613-521-9215; courriel : patty@canadianstrokenetwork.ca) 
Annexe 1: Membres du Groupe de travail sur les pratiques optimales et les normes et du Groupe de travail sur l'information et l'évaluation de la Stratégie canadienne de l'AVC (partie 1).

\begin{tabular}{|c|c|c|c|}
\hline Nom & Poste & Établissement ou organisation & Intérêts concurrents \\
\hline \multicolumn{4}{|c|}{ Groupe de travail de la plate-forme nationale Pratiques optimales et normes } \\
\hline $\begin{array}{l}\text { D' Stephen Phillips* } \\
\text { (coprésident) }\end{array}$ & Directeur, Programme de l'ACV aigu & $\begin{array}{l}\text { Queen Elizabeth II Health Sciences } \\
\text { Centre, Halifax (N.-É.) }\end{array}$ & Aucun déclaré. \\
\hline $\begin{array}{l}\mathrm{M}^{\mathrm{me}} \text { Alison McDonald } \\
\text { (coprésidente) }\end{array}$ & $\begin{array}{l}\text { Physiothérapeute, Nova Scotia } \\
\text { Rehabilitation Centre }\end{array}$ & $\begin{array}{l}\text { Queen Elizabeth II Health Sciences } \\
\text { Centre, Halifax (N.-É.) }\end{array}$ & Aucun déclaré. \\
\hline D' Mark Bayley* & $\begin{array}{l}\text { Physiatre et directeur médical, } \\
\text { Programme de réadaptation } \\
\text { neurologique }\end{array}$ & $\begin{array}{l}\text { Toronto Rehabilitation Institute, } \\
\text { Toronto (Ont.) }\end{array}$ & Aucun déclaré. \\
\hline D' Alan Bell & Médecin de famille & $\begin{array}{l}\text { Collège des médecins de famille du } \\
\text { Canada, Toronto (Ont.) }\end{array}$ & $\begin{array}{l}\text { Subventions et honoraires de chercheur, } \\
\text { de consultant et de conférencier, de } \\
\text { Sanofi-Aventis, Bristol-Myers Squibb, } \\
\text { Bayer et AstraZeneca. }\end{array}$ \\
\hline $\mathrm{M}^{\mathrm{me}}$ Laurie Cameron & $\begin{array}{l}\text { Coordonnatrice de programme et } \\
\text { adjointe de direction }\end{array}$ & $\begin{array}{l}\text { Stratégie canadienne sur l'AVC, } \\
\text { Ottawa (Ont.) }\end{array}$ & Aucun déclaré. \\
\hline $\mathrm{M}^{\mathrm{me}}$ Nancy Cooper & $\begin{array}{l}\text { Directrice, Politiques et } \\
\text { Perfectionnement professionnel }\end{array}$ & $\begin{array}{l}\text { Ontario Long-Term Care Association, } \\
\text { Markham (Ont.) }\end{array}$ & Aucun déclaré. \\
\hline $\mathrm{M}^{\mathrm{me}}$ Bev Culham & Gestionnaire de projet & $\begin{array}{l}\text { Alberta Provincial Stroke Strategy, } \\
\text { Calgary (Alb.) }\end{array}$ & Aucun déclaré. \\
\hline D' lan Graham & $\begin{array}{l}\text { Vice-président, Application des } \\
\text { connaissances }\end{array}$ & $\begin{array}{l}\text { Instituts de recherche en santé du } \\
\text { Canada, Ottawa (Ont.) }\end{array}$ & Aucun déclaré. \\
\hline D' Gordon Gubitz & $\begin{array}{l}\text { Neurologue, Unité de l'AVC aigu et } \\
\text { Clinique externe neurovasculaire }\end{array}$ & $\begin{array}{l}\text { Queen Elizabeth II Health Sciences } \\
\text { Centre, Halifax (N.-É.) }\end{array}$ & Aucun déclaré. \\
\hline $\begin{array}{l}\mathrm{M}^{\mathrm{me}} \text { Mary Elizabeth } \\
\text { Harriman }\end{array}$ & Directrice générale associée & $\begin{array}{l}\text { Fondation des maladies du cœur du } \\
\text { Canada, Ottawa (Ont.) }\end{array}$ & Aucun déclaré. \\
\hline $\mathrm{M}^{\mathrm{me}}$ Chelsea Hellings* & Coordonnatrice de la recherche & $\begin{array}{l}\text { Stratégie canadienne de l'AVC, } \\
\text { Toronto (Ont.) }\end{array}$ & Aucun déclaré. \\
\hline $\mathrm{M}^{\mathrm{me}}$ Shelley Irvine-Day & Pathologiste du langage & Deer Lodge Centre, Winnipeg (Man.) & Aucun déclaré. \\
\hline $\mathrm{M}^{\mathrm{me}}$ Nina I. Jetha & $\begin{array}{l}\text { Gestionnaire principale de } \\
\text { programme, Direction de la } \\
\text { promotion de la santé et de la } \\
\text { prévention des maladies chroniques }\end{array}$ & $\begin{array}{l}\text { Agence de la santé publique du } \\
\text { Canada, Ottawa (Ont.) }\end{array}$ & Aucun déclaré. \\
\hline $\mathrm{M}^{\mathrm{me}}$ Katie Lafferty & Directrice générale & $\begin{array}{l}\text { Réseau canadien contre les accidents } \\
\text { cérébrovasculaires, Ottawa (Ont.) }\end{array}$ & Aucun déclaré. \\
\hline D' Sylvain Lanthier & Neurologue spécialisé en AVC & $\begin{array}{l}\text { Centre hospitalier de l'Université de } \\
\text { Montréal, Montréal (QC) }\end{array}$ & $\begin{array}{l}\text { Honoraires de conférencier et de } \\
\text { participant à des conseils consultatifs (ne } \\
\text { dépassant jamais } 10000 \text { \$) de Pfizer, } \\
\text { Sanofi-Aventis, Bristol-Myers Squibb, } \\
\text { Boehringer Ingelheim, Merck-Frosst et } \\
\text { Servier. Des frais de voyage reliés à ces } \\
\text { activités ont également été payés. }\end{array}$ \\
\hline $\begin{array}{l}\mathrm{M}^{\mathrm{me}} \text { Patrice Lindsay, } \mathrm{PhD}^{*} \\
\text { (directrice des membres } \\
\text { du personnel) }\end{array}$ & Spécialiste, Rendement et normes & $\begin{array}{l}\text { Réseau canadien contre les accidents } \\
\text { cérébrovasculaires, Toronto (Ont.) }\end{array}$ & Aucun déclaré. \\
\hline $\mathrm{M}^{\mathrm{me}}$ Michelle McKay & $\begin{array}{l}\text { Infirmière praticienne spécialisée en } \\
\text { neurologie }\end{array}$ & $\begin{array}{l}\text { Queen Elizabeth II Health Sciences } \\
\text { Centre, Halifax (N.-É.) }\end{array}$ & Aucun déclaré. \\
\hline $\mathrm{M}^{\mathrm{me}}$ Janel Nadeau & Représentante des patients & $\begin{array}{l}\text { Stroke Recovery Association, Calgary } \\
\text { (Alb.) }\end{array}$ & Aucun déclaré. \\
\hline $\mathrm{M}^{\mathrm{me}}$ Louise Nichol & $\begin{array}{l}\text { Gestionnaire d'équipe } \\
\text { communautaire, Programme de soins } \\
\text { à domicile }\end{array}$ & $\begin{array}{l}\text { Community Stroke Care Service, } \\
\text { Winnipeg (Man.) }\end{array}$ & Aucun déclaré. \\
\hline $\mathrm{M}^{\mathrm{me}}$ Christina O'Callaghan & $\begin{array}{l}\text { Gestionnaire, Programme régional de } \\
\text { l'AVC }\end{array}$ & $\begin{array}{l}\text { London Health Sciences Centre, } \\
\text { London (Ont.) }\end{array}$ & Aucun déclaré. \\
\hline D' Tejal Patel & Pharmacienne & St. Michael's Hospital, Toronto (Ont.) & Aucun déclaré. \\
\hline
\end{tabular}


Annexe 1 : Membres du Groupe de travail sur les pratiques optimales et les normes et du Groupe de travail sur l'information et l'évaluation de la Stratégie canadienne de l'AVC (partie 2).

\begin{tabular}{|c|c|c|c|}
\hline Nom & Poste & Établissement ou organisation & Intérêts concurrents \\
\hline \multicolumn{4}{|c|}{ Groupe de travail de la plate-forme nationale Pratiques optimales et normes (suite) } \\
\hline$M^{m e}$ Elizabeth Woodbury* & Directrice générale & $\begin{array}{l}\text { Stratégie canadienne de l'AVC, } \\
\text { Ottawa (Ont.) }\end{array}$ & Aucun déclaré. \\
\hline \multicolumn{4}{|c|}{ Tribune nationale du Groupe de travail sur l'information et l'évaluation } \\
\hline \multirow[t]{2}{*}{$\begin{array}{l}\text { D' Michael Hill* } \\
\text { (coprésident) }\end{array}$} & $\begin{array}{l}\text { Directeur, Unité de l'AVC, Calgary } \\
\text { Stroke Program }\end{array}$ & $\begin{array}{l}\text { Foothills Medical Centre, Calgary } \\
\text { (Alb.) }\end{array}$ & $\begin{array}{l}\text { Soutien pour des essais, de Baxter, Merck } \\
\text { et Hoffmann-LaRoche. }\end{array}$ \\
\hline & & & $\begin{array}{l}\text { Expert-conseil auprès de Genentech Ltd., } \\
\text { Vanalis Group Ltd., Sanofi, Portola Inc., } \\
\text { NONO Inc., Stem Cell Therapeutics, } \\
\text { Hoffmann-LaRoche. Honoraires de } \\
\text { conférencier de toutes les sociétés } \\
\text { susmentionnées, plus de Boehringer } \\
\text { Ingelheim et Bristol-Myers Squibb Canada. }\end{array}$ \\
\hline $\begin{array}{l}\mathrm{M}^{\mathrm{me}} \text { Patrice Lindsay, PhD* } \\
\text { (coprésidente) }\end{array}$ & Spécialiste, Rendement et normes & $\begin{array}{l}\text { Réseau canadien contre les accidents } \\
\text { cérébrovasculaires, Toronto (Ont.) }\end{array}$ & Aucun déclaré. \\
\hline $\mathrm{M}^{\mathrm{me}}$ Asako Bienek & $\begin{array}{l}\text { Analyste, Division de la surveillance, } \\
\text { Centre de prévention et de surveillance } \\
\text { des maladies chroniques }\end{array}$ & $\begin{array}{l}\text { Agence de la santé publique du } \\
\text { Canada, Ottawa (Ont.) }\end{array}$ & Aucun déclaré. \\
\hline D' Robert Côté & $\begin{array}{l}\text { Neurologue spécialisé en AVC et } \\
\text { président, Comité consultatif sur les } \\
\text { politiques de recherche et la } \\
\text { planification, Fondation des maladies } \\
\text { du cœur du Canada }\end{array}$ & $\begin{array}{l}\text { Hôpital Général de Montréal, } \\
\text { Montréal (Qc) }\end{array}$ & $\begin{array}{l}\text { Honoraires de conférencier de Aventis- } \\
\text { Sanofi, Boehringer-Ingelheim, Merck, } \\
\text { Solvay; remboursement de frais de voyage } \\
\text { pour des réunions de consultation avec } \\
\text { Pfizer. }\end{array}$ \\
\hline $\begin{array}{l}\mathrm{M}^{\text {me }} \text { Mary Elizabeth } \\
\text { Harriman }\end{array}$ & Directrice générale associée & $\begin{array}{l}\text { Fondation des maladies du cœur du } \\
\text { Canada, Ottawa (Ont.) }\end{array}$ & Aucun déclaré. \\
\hline D' Tom Jeerakathil & Neurologue spécialisé en AVC & $\begin{array}{l}\text { Department of Medicine, University } \\
\text { of Alberta, Edmonton (Alb.) }\end{array}$ & $\begin{array}{l}\text { Participation à un comité consultatif pour } \\
\text { Novo Nordisk, qui s'est réuni une fois en } \\
2007 .\end{array}$ \\
\hline D' Moira Kapral & Interniste générale et chercheure & $\begin{array}{l}\text { Toronto General Hospital, University } \\
\text { Health Network, Toronto (Ont.) }\end{array}$ & yAucun déclaré. \\
\hline $\mathrm{M}^{\mathrm{me}}$ Katie Lafferty & Directrice générale & $\begin{array}{l}\text { Réseau canadien contre les accidents } \\
\text { cérébrovasculaires, Ottawa (Ont.) }\end{array}$ & Aucun déclaré. \\
\hline D' Grace Warner & Chercheure & $\begin{array}{l}\text { Atlantic Health Promotion Research } \\
\text { Centre, Université Dalhousie, Halifax } \\
\text { (N.-É.) }\end{array}$ & Aucun déclaré. \\
\hline $\mathrm{M}^{\mathrm{me}}$ Elizabeth Woodbury* & Directrice générale & $\begin{array}{l}\text { Stratégie canadienne de l'AVC, } \\
\text { Ottawa (Ont.) }\end{array}$ & Aucun déclaré. \\
\hline
\end{tabular}

* Membres du Groupe de rédaction sur les pratiques optimales et les normes. 
Annexe 2 : Membres des groupes de travail des Recommandations canadiennes pour les pratiques optimales de soins de l'AVC (partie 1).

\begin{tabular}{|c|c|c|c|}
\hline Nom & Poste & Établissement ou organisation & Intérêts concurrents \\
\hline \multicolumn{4}{|c|}{ Groupe de travail sur la prévention } \\
\hline $\begin{array}{l}\text { D' Demetrios Sahlas } \\
\text { (président) }\end{array}$ & Neurologue spécialisé en AVC & $\begin{array}{l}\text { Hamilton Health Sciences, Hamilton } \\
\text { (Ont.) }\end{array}$ & $\begin{array}{l}\text { Honoraires de conférencier il y a deux } \\
\text { ans de Pfizer au sujet de l'essai sur la } \\
\text { prévention de l'AVC par réduction } \\
\text { agressive des niveaux de cholestérol } \\
\text { (Stroke Prevention by Aggressive } \\
\text { Reduction in Cholesterol Levels - } \\
\text { SPARCL). }\end{array}$ \\
\hline D' Alan Bell & Médecin de famille & $\begin{array}{l}\text { Collège des médecins de famille du } \\
\text { Canada, Toronto (Ont.) }\end{array}$ & $\begin{array}{l}\text { Subventions et honoraires de chercheur, } \\
\text { consultant et conférencier, de Sanofi- } \\
\text { Aventis, Bristol-Myers Squibb, Bayer et } \\
\text { AstraZeneca. }\end{array}$ \\
\hline $\mathrm{M}^{\mathrm{me}}$ Eryka Hailey & $\begin{array}{l}\text { Infirmière spécialisée en prévention de l'AVC } \\
\text { et chercheure }\end{array}$ & Foothills Medical Centre, Calgary (Alb.) & ) Aucun déclaré. \\
\hline $\mathrm{M}^{\mathrm{me}}$ Chelsea Hellings & Coordonnatrice de la recherche & $\begin{array}{l}\text { Stratégie canadienne de l'AVC, } \\
\text { Toronto (Ont.) }\end{array}$ & Aucun déclaré. \\
\hline D'Mark Hudon & Neuroradiologiste & Foothills Medical Centre, Calgary (Alb.) & ) Aucun déclaré. \\
\hline D'Tom Jeerakathil & Neurologue spécialisé en AVC & $\begin{array}{l}\text { Department of Medicine, University of } \\
\text { Alberta, Edmonton (Alb.) }\end{array}$ & $\begin{array}{l}\text { f Participation à un comité consultatif } \\
\text { pour Novo Nordisk, qui s'est réuni une } \\
\text { fois en } 2007 \text {. }\end{array}$ \\
\hline $\mathbf{M}^{\mathrm{me}}$ Linda Kelloway & Experte-conseil en éducation sur l'AVC & $\begin{array}{l}\text { Fondation des maladies du cœur du } \\
\text { Canada, Toronto (Ont.) }\end{array}$ & Aucun déclaré. \\
\hline $\begin{array}{l}\mathrm{M}^{\mathrm{me}} \text { Patrice Lindsay, } \\
\mathrm{PhD}\end{array}$ & Spécialiste, Rendement et normes & $\begin{array}{l}\text { Réseau canadien contre les accidents } \\
\text { cérébrovasculaires, Toronto (Ont.) }\end{array}$ & Aucun déclaré. \\
\hline $\mathrm{D}^{\prime}$ Tejal Patel & Pharmacienne & St. Michael's Hospital, Toronto (Ont.) & Aucun déclaré. \\
\hline D' Stephen Phillips & Directeur, Programme de l'AVC aigu & $\begin{array}{l}\text { Queen Elizabeth II Health Sciences } \\
\text { Centre, Halifax (N.-É.) }\end{array}$ & Aucun déclaré. \\
\hline$D^{r}$ Jon Witt & $\begin{array}{l}\text { Directeur, Soins de l'AVC aigu, Département } \\
\text { de médecine d'urgence }\end{array}$ & $\begin{array}{l}\text { Royal University Hospital, Saskatoon } \\
\text { (Sask.) }\end{array}$ & $\begin{array}{l}\text { Honoraires de conférencier et frais de } \\
\text { déplacement pour un programme } \\
\text { d'éducation (symposium satellite de } \\
\text { l'Association canadienne des médecins } \\
\text { d'urgence du Canada en 2007); réunion } \\
\text { de trois jours d'un conseil consultatif de } \\
\text { Roche en } 2007 \text {. }\end{array}$ \\
\hline \multicolumn{4}{|c|}{ Groupe de travail sur les soins préhospitaliers } \\
\hline $\begin{array}{l}\text { M. Pierre Poirier } \\
\text { (président) }\end{array}$ & Directeur général & $\begin{array}{l}\text { Association des Paramédics du } \\
\text { Canada, Ottawa (Ont.) }\end{array}$ & Aucun déclaré. \\
\hline $\mathrm{M}^{\mathrm{me}}$ Chelsea Hellings & Coordonnatrice de la recherche & $\begin{array}{l}\text { Stratégie canadienne de l'AVC, } \\
\text { Toronto (Ont.) }\end{array}$ & Aucun déclaré. \\
\hline $\mathrm{M}^{\mathrm{me}}$ Linda Kelloway & Experte-conseil en éducation sur I'AVC & $\begin{array}{l}\text { Fondation des maladies du cœur du } \\
\text { Canada, Toronto (Ont.) }\end{array}$ & Aucun déclaré. \\
\hline $\begin{array}{l}\mathrm{M}^{\mathrm{me}} \text { Brenda } \\
\text { Kwiatkowski }\end{array}$ & $\begin{array}{l}\text { Coordonnatrice, Clinique de prévention de } \\
\text { l'AVC }\end{array}$ & $\begin{array}{l}\text { Saskatoon Health Region, Saskatoon } \\
\text { (Sask.) }\end{array}$ & Aucun déclaré. \\
\hline $\begin{array}{l}M^{m e} \text { Patrice Lindsay, } \\
\text { PhD }\end{array}$ & Spécialiste, Rendement et normes & $\begin{array}{l}\text { Réseau canadien contre les accidents } \\
\text { cérébrovasculaires, Toronto (Ont.) }\end{array}$ & Aucun déclaré. \\
\hline M. Mike Nolan & $\begin{array}{l}\text { Chef des services d'urgence, Services des } \\
\text { paramédics }\end{array}$ & $\begin{array}{l}\text { County of Renfrew Paramedic Service, } \\
\text { Renfrew, Ont. }\end{array}$ & Aucun déclaré. \\
\hline $\mathrm{M}^{\mathrm{me}}$ Chris O'Callaghan & $\begin{array}{l}\text { Coordonnatrice, Programme régional sur } \\
\text { I'AVC }\end{array}$ & $\begin{array}{l}\text { London Health Sciences Centre, } \\
\text { London (Ont.) }\end{array}$ & Aucun déclaré. \\
\hline D' Andrew Travers & $\begin{array}{l}\text { Directeur médical provincial, Services } \\
\text { d'urgence en santé }\end{array}$ & Halifax (N.-É.) & $\begin{array}{l}\text { Honoraires de conférencier de Roche au } \\
\text { sujet des services médicaux d'urgence et } \\
\text { des soins en cas de STEMl et d'AVC. }\end{array}$ \\
\hline D' Karen Wanger & $\begin{array}{l}\text { Directrice médicale, Mainland } \\
\text { Administration }\end{array}$ & $\begin{array}{l}\text { British Columbia Ambulance Service, } \\
\text { Vancouver (C.-B.) }\end{array}$ & Aucun déclaré. \\
\hline
\end{tabular}


Annexe 2 : Membres des groupes de travail des Recommandations canadiennes pour les pratiques optimales de soins de l'AVC (partie 2).

\begin{tabular}{|c|c|c|c|}
\hline Nom & Poste & Établissement ou organisation & Intérêts concurrents \\
\hline \multicolumn{4}{|c|}{ Groupe de travail sur les soins préhospitaliers (suite) } \\
\hline D' Jon Witt & $\begin{array}{l}\text { Directeur, Soins de l'AVC aigu, } \\
\text { Département de médecine d'urgence }\end{array}$ & $\begin{array}{l}\text { Royal University Hospital, Saskatoon } \\
\text { (Sask.) }\end{array}$ & $\begin{array}{l}\text { Honoraires de conférencier et frais de } \\
\text { déplacement pour un programme } \\
\text { d'éducation (symposium satellite de } \\
\text { l'Association canadienne des médecins } \\
\text { d'urgence du Canada en 2007); réunion } \\
\text { de trois jours d'un conseil consultatif de } \\
\text { Roche en } 2007 \text {. }\end{array}$ \\
\hline \multicolumn{4}{|c|}{ Groupe de travail sur le soin de l'AVC aigu } \\
\hline D' Gordon Gubitz (Chair) & $\begin{array}{l}\text { Neurologue, Unité de l'ACD aigu et } \\
\text { Clinique neurovasculaire externe }\end{array}$ & $\begin{array}{l}\text { Queen Elizabeth II Health Sciences } \\
\text { Centre, Halifax (N.-É.) }\end{array}$ & Aucun déclaré. \\
\hline $\mathrm{M}^{\mathrm{me}}$ Cindy Bolton & $\begin{array}{l}\text { Gestionnaire de programme et } \\
\text { membre du Comité directeur de la } \\
\text { Stratégie canadienne de l'AVC }\end{array}$ & $\begin{array}{l}\text { Kingston General Hospital, Kingston } \\
\text { (Ont.) }\end{array}$ & Aucun déclaré. \\
\hline $\mathrm{M}^{\mathrm{me}}$ Margaret Grant & $\begin{array}{l}\text { Coordonnatrice, Éducation à la } \\
\text { réadaptation }\end{array}$ & $\begin{array}{l}\text { Alberta Provincial Stroke Strategy, } \\
\text { Calgary (Alb.) }\end{array}$ & Aucun déclaré. \\
\hline D'Teri Green & $\begin{array}{l}\text { Coordonnatrice, Calgary Stroke } \\
\text { Program }\end{array}$ & \multicolumn{2}{|c|}{ Foothills Medical Centre, Calgary (Alb.) Aucun déclaré. } \\
\hline $\mathrm{M}^{\mathrm{me}}$ Chelsea Hellings & Coordonnatrice de la recherche & $\begin{array}{l}\text { Stratégie canadienne de l'AVC, } \\
\text { Toronto (Ont.) }\end{array}$ & Aucun déclaré. \\
\hline $\mathrm{M}^{\mathrm{me}}$ Shelly Irvine-Day & Pathologiste, troubles du langage & Deer Lodge Centre, Winnipeg (Man.) & Aucun déclaré. \\
\hline $\mathrm{M}^{\mathrm{me}}$ Joanne Lee & Diététiste & Calgary Health Region, Calgary (Alb.) & Aucun déclaré. \\
\hline $\mathrm{M}^{\mathrm{me}}$ Linda Kelloway & Experte-conseil en AVC & $\begin{array}{l}\text { Fondation des maladies du cœur du } \\
\text { Canada, Toronto (Ont.) }\end{array}$ & Aucun déclaré. \\
\hline $\mathrm{M}^{\mathrm{me}}$ Patrice Lindsay, PhD & Spécialiste, Rendement et normes & $\begin{array}{l}\text { Réseau canadien contre les accidents } \\
\text { cérébrovasculaires, Toronto (Ont.) }\end{array}$ & Aucun déclaré. \\
\hline $\mathrm{M}^{\mathrm{me}}$ Alison McDonald & $\begin{array}{l}\text { Physiothérapeute, Nova Scotia } \\
\text { Rehabilitation Centre }\end{array}$ & $\begin{array}{l}\text { Queen Elizabeth II Health Sciences } \\
\text { Centre, Halifax (N.-É.) }\end{array}$ & Aucun déclaré. \\
\hline M $^{\text {me }}$ Michelle McKay & $\begin{array}{l}\text { Infirmière praticienne spécialisée en } \\
\text { neurologie }\end{array}$ & $\begin{array}{l}\text { Queen Elizabeth II Health Sciences } \\
\text { Centre, Halifax (N.-É.) }\end{array}$ & Aucun déclaré. \\
\hline D' Tejal Patel & Pharmacienne & St. Michael's Hospital, Toronto (Ont.) & Aucun déclaré. \\
\hline D' Michael Sigal & Dentiste en chef & Mount Sinai Hospital, Toronto (Ont.) & Aucun déclaré. \\
\hline \multicolumn{4}{|c|}{ Groupe de travail sur la démence consécutive à l'AVC } \\
\hline D' Sandra Black (Chair) & Chaire Brill de neurologie & $\begin{array}{l}\text { University of Toronto, Sunnybrook } \\
\text { Health Sciences Centre, Toronto (Ont.) }\end{array}$ & $\begin{array}{l}\text { Consultation à l'occasion, y compris } \\
\text { participation à un conseil consultatif, } \\
\text { selon mes disponibilités, pour Novartis, } \\
\text { Pfizer, Eisai, Janssen-Ortho, Lundbeck, } \\
\text { Glaxo-Smith-Kline, Myriad, EBIX (ne } \\
\text { dépassant pas } 10000 \$ \text { par société). } \\
\text { Honoraires de conférencier de Novartis, } \\
\text { Pfizer, JanssenOrtho, Lundbeck, Myriad. }\end{array}$ \\
\hline D' Mark Bayley & $\begin{array}{l}\text { Physiatre et directeur médical, } \\
\text { Programme de réadaptation } \\
\text { neurologique }\end{array}$ & $\begin{array}{l}\text { Toronto Rehabilitation Institute, } \\
\text { Toronto (Ont.) }\end{array}$ & Aucun déclaré. \\
\hline
\end{tabular}


Annexe 2 : Membres des groupes de travail des Recommandations canadiennes pour les pratiques optimales de soins de l'AVC (partie 3).

\begin{tabular}{|c|c|c|c|}
\hline Nom & Poste & Établissement ou organisation & Intérêts concurrents \\
\hline \multicolumn{4}{|c|}{ Groupe de travail sur la démence consécutive à l'ACV (suite) } \\
\hline D' Gail Eskes & $\begin{array}{l}\text { Professeure agrégée de psychiatrie et } \\
\text { psychologie }\end{array}$ & Dalhousie University, Halifax (N.-É.) & Aucun déclaré. \\
\hline D' Antoine Hakim & $\begin{array}{l}\text { Chef de la direction et directeur } \\
\text { scientifique }\end{array}$ & $\begin{array}{l}\text { Réseau canadien contre les accidents } \\
\text { cérébrovasculaires, Ottawa (Ont.) }\end{array}$ & Aucun déclaré. \\
\hline $\mathrm{M}^{\mathrm{me}}$ Chelsea Hellings & Coordonnatrice de la recherche & $\begin{array}{l}\text { Stratégie canadienne de l'AVC, } \\
\text { Toronto (Ont.) }\end{array}$ & Aucun déclaré. \\
\hline $\mathrm{M}^{\mathrm{me}}$ Shelley Irvine-Day & Pathologiste, Troubles du langage & Deer Lodge Centre, Winnipeg (Man.) & Aucun déclaré. \\
\hline $\mathrm{M}^{\mathrm{me}}$ Patrice Lindsay, PhD & Spécialiste, Rendement et normes & $\begin{array}{l}\text { Réseau canadien contre les accidents } \\
\text { cérébrovasculaires, Toronto (Ont.) }\end{array}$ & Aucun déclaré. \\
\hline $\mathrm{M}^{\mathrm{me}}$ Louise Nichol & $\begin{array}{l}\text { Gestionnaire de l'équipe } \\
\text { communautaire, Programme de soins } \\
\text { à domicile }\end{array}$ & $\begin{array}{l}\text { Community Stroke Care Service, } \\
\text { Winnipeg (Man.) }\end{array}$ & Aucun déclaré. \\
\hline D' David L. Nyenhuis & $\begin{array}{l}\text { Département de neurologie et } \\
\text { réadaptation }\end{array}$ & $\begin{array}{l}\text { University of Illinois, Chicago Center } \\
\text { for Stroke Research, Chicago, IL }\end{array}$ & $\begin{array}{l}\text { Honoraires de conférencier et de } \\
\text { consultant de Eisai-Pfizer. }\end{array}$ \\
\hline $\mathrm{M}^{\mathrm{me}}$ Jill Moats & $\begin{array}{l}\text { Éducatrice régionale, Programme de } \\
\text { réadaptation et gériatrie }\end{array}$ & $\begin{array}{l}\text { Winnipeg Regional Health Authority, } \\
\text { Winnipeg (Man.) }\end{array}$ & Aucun déclaré. \\
\hline D' Stephen Phillips & Directeur, Programme de l'AVC aigu & $\begin{array}{l}\text { Queen Elizabeth II Health Sciences } \\
\text { Centre, Halifax (N.-É.) }\end{array}$ & Aucun déclaré. \\
\hline D' Kenneth Rockwood & Département de médecine & $\begin{array}{l}\text { Queen Elizabeth II Health Sciences } \\
\text { Centre, Halifax (N.-É.) }\end{array}$ & $\begin{array}{l}\text { Expert conseil auprès de Pfizer, } \\
\text { JansenOrtho, Novartis. Honoraires de } \\
\text { conférencier de Novartis. }\end{array}$ \\
\hline$D^{\prime}$ Jon Ween & $\begin{array}{l}\text { Directeur, Clinique de l'AVC et de la } \\
\text { cognition }\end{array}$ & $\begin{array}{l}\text { Baycrest Centre for Geriatric Care, } \\
\text { Toronto (Ont.) }\end{array}$ & Aucun déclaré. \\
\hline \multicolumn{4}{|c|}{ Groupe de travail sur la réadaptation } \\
\hline D' Mark Bayley (Chair) & $\begin{array}{l}\text { Physiatre et directeur médical, } \\
\text { Programme de réadaptation } \\
\text { neurologique }\end{array}$ & $\begin{array}{l}\text { Toronto Rehabilitation Institute, } \\
\text { Toronto (Ont.) }\end{array}$ & Aucun déclaré. \\
\hline $\mathrm{M}^{\mathrm{me}}$ Barb Ansley & $\begin{array}{l}\text { Coordonnatrice, Recherche en } \\
\text { réadaptation et évaluation de } \\
\text { programme }\end{array}$ & $\begin{array}{l}\text { Hamilton Health Sciences, Hamilton } \\
\text { (Ont.) }\end{array}$ & Aucun déclaré. \\
\hline $\mathrm{M}^{\mathrm{me}}$ Nancy Boaro & $\begin{array}{l}\text { Chef d'équipe en pratique avancée, } \\
\text { Programme de réadaptation } \\
\text { neurologique }\end{array}$ & $\begin{array}{l}\text { Toronto Rehabilitation Institute, } \\
\text { Toronto (Ont.) }\end{array}$ & Aucun déclaré. \\
\hline $\mathrm{M}^{\mathrm{me}}$ Jenn Fearn & $\begin{array}{l}\text { Coordonnatrice, Réadaptation, } \\
\text { Réseau du Nord-est ontarien pour } \\
\text { I'AVC }\end{array}$ & $\begin{array}{l}\text { Sudbury Regional Hospital, Sudbury, } \\
\text { Ont. }\end{array}$ & Aucun déclaré. \\
\hline $\mathrm{M}^{\mathrm{me}}$ Chelsea Hellings & Coordonnatrice, Recherche & $\begin{array}{l}\text { Stratégie canadienne de l'AVC, } \\
\text { Toronto (Ont.) }\end{array}$ & Aucun déclaré. \\
\hline $\mathrm{M}^{\mathrm{me}}$ Shelley Irvine-Day & Pathologiste, Troubles du langage & Deer Lodge Centre, Winnipeg (Man.) & Aucun déclaré. \\
\hline $\mathrm{M}^{\mathrm{me}}$ Patrice Lindsay, PhD & Spécialiste, Rendement et normes & $\begin{array}{l}\text { Réseau canadien contre les accidents } \\
\text { cérébrovasculaires, Toronto (Ont.) }\end{array}$ & Aucun déclaré. \\
\hline $\mathrm{M}^{\mathrm{me}}$ Alison McDonald & $\begin{array}{l}\text { Physiothérapeute, Nova Scotia } \\
\text { Rehabilitation Centre }\end{array}$ & $\begin{array}{l}\text { Queen Elizabeth II Health Sciences } \\
\text { Centre, Halifax (N.-É.) }\end{array}$ & Aucun déclaré. \\
\hline $\mathrm{M}^{\mathrm{me}}$ Louise Nichol & $\begin{array}{l}\text { Gestionnaire de l'équipe } \\
\text { communautaire, Programme de soins } \\
\text { à domicile }\end{array}$ & $\begin{array}{l}\text { Community Stroke Care Service, } \\
\text { Winnipeg (Man.) }\end{array}$ & Aucun déclaré. \\
\hline
\end{tabular}


Annexe 2 : Membres des groupes de travail des Recommandations canadiennes pour les pratiques optimales de soins de l'AVC (partie 4).

\begin{tabular}{|c|c|c|c|}
\hline Nom & Poste & Établissement ou organisation & Intérêts concurrents \\
\hline \multicolumn{4}{|c|}{ Groupe de travail sur la réadaptation (suite) } \\
\hline$D^{\prime}$ Robert Teasell & Physiatre & Parkwood Hospital, London (Ont.) & $\begin{array}{l}\text { Dépenses de voyage pour des } \\
\text { présentations à Winnipeg, } \\
\text { Toronto, Ottawa et St. John's. }\end{array}$ \\
\hline \multicolumn{4}{|c|}{ Groupe de travail sur l'éducation des patients et des familles } \\
\hline $\begin{array}{l}\mathrm{M}^{\mathrm{me}} \text { Linda Kelloway } \\
\text { (présidente) }\end{array}$ & Experte-conseil, Éducation sur l'AVC & $\begin{array}{l}\text { Fondation des maladies du cœur du } \\
\text { Canada, Toronto (Ont.) }\end{array}$ & Aucun déclaré. \\
\hline $\mathrm{M}^{\mathrm{me}}$ Jill Cameron & $\begin{array}{l}\text { Professeure agrégée, Département de } \\
\text { sciences de l'ergothérapie }\end{array}$ & University of Toronto, Toronto (Ont.) & Aucun déclaré. \\
\hline $\mathrm{M}^{\mathrm{me}}$ Patti Gallagher & $\begin{array}{l}\text { Infirmière clinique spécialisée, } \\
\text { Neuroscience }\end{array}$ & $\begin{array}{l}\text { Saint John Regional Hospital, Saint } \\
\text { John (N.-B.) }\end{array}$ & Aucun déclaré. \\
\hline $\mathrm{M}^{\mathrm{me}}$ Chelsea Hellings & Coordonnatrice de la recherche & $\begin{array}{l}\text { Stratégie canadienne de l'AVC, } \\
\text { Toronto (Ont.) }\end{array}$ & Aucun déclaré. \\
\hline $\mathrm{M}^{\mathrm{me}}$ Samantha Li & $\begin{array}{l}\text { Gestionnaire associée principale, } \\
\text { Information sur la santé }\end{array}$ & $\begin{array}{l}\text { Fondation des maladies du cœur de } \\
\text { I'Ontario, Toronto (Ont.) }\end{array}$ & Aucun déclaré. \\
\hline $\mathrm{M}^{\mathrm{me}}$ Patrice Lindsay, $\mathrm{PhD}$ & Spécialiste, Rendement et normes & $\begin{array}{l}\text { Réseau canadien contre les accidents } \\
\text { cérébrovasculaires, Toronto (Ont.) }\end{array}$ & Aucun déclaré. \\
\hline $\mathrm{M}^{\mathrm{me}}$ Alison McDonald & $\begin{array}{l}\text { Physiothérapeute, Nova Scotia } \\
\text { Rehabilitation Centre }\end{array}$ & $\begin{array}{l}\text { Queen Elizabeth II Health Sciences } \\
\text { Centre, Halifax (N.-É.) }\end{array}$ & Aucun déclaré. \\
\hline M. Frank Nieboer & Président sortant & $\begin{array}{l}\text { Alberta Stroke Recovery Association, } \\
\text { Calgary (Alb.) }\end{array}$ & Aucun déclaré. \\
\hline $\mathrm{M}^{\mathrm{me}}$ Jody Yuzik & Chef d'équipe, Gestion des soins & $\begin{array}{l}\text { GF Strong Rehabilitation Centre, } \\
\text { Vancouver (C.-B.) }\end{array}$ & Aucun déclaré. \\
\hline \multicolumn{4}{|c|}{ Groupe de travail sur la sensibilisation du public } \\
\hline $\begin{array}{l}\mathrm{M}^{\mathrm{me}} \text { Patrice Lindsay, PhD } \\
\text { (présidente) }\end{array}$ & Spécialiste, Rendement et normes & $\begin{array}{l}\text { Réseau canadien contre les accidents } \\
\text { cérébrovasculaires, Toronto (Ont.) }\end{array}$ & Aucun déclaré. \\
\hline $\mathrm{M}^{\mathrm{me}}$ Heather Rourke & Directrice des communications & $\begin{array}{l}\text { Fondation des maladies du cœur du } \\
\text { Canada, Ottawa (Ont.) }\end{array}$ & Aucun déclaré. \\
\hline $\mathrm{M}^{\mathrm{me}}$ Chelsea Hellings & Coordonnatrice de la recherche & $\begin{array}{l}\text { Stratégie canadienne de l'AVC, } \\
\text { Toronto (Ont.) }\end{array}$ & Aucun déclaré. \\
\hline $\mathrm{M}^{\mathrm{me}}$ Corinne Hodgson & Experte-conseil & Toronto (Ont.) & Aucun déclaré. \\
\hline $\mathrm{M}^{\mathrm{me}}$ Brenda Kwiatkowski & $\begin{array}{l}\text { Membre, National Stroke Nursing } \\
\text { Council }\end{array}$ & Saskatoon Health, Saskatoon (Sask.) & Aucun déclaré. \\
\hline M. Craig Pierre & Gestionnaire général & Island EMS, Charlottetown (Î.-P.-É.) & Aucun déclaré. \\
\hline M. Pierre Poirier & Directeur général & $\begin{array}{l}\text { Association des Paramédics du } \\
\text { Canada, Ottawa (Ont.) }\end{array}$ & Aucun déclaré. \\
\hline D' Eli Segal & Médecin attitré, Service d'urgence & $\begin{array}{l}\text { Hôpital juif Sir-Mortimer-B.-Davis, } \\
\text { Montréal (QC) }\end{array}$ & Aucun déclaré. \\
\hline
\end{tabular}

